\title{
VIII. Organisatorische Veränderungen im Verhältnis zwischen SED und NVA 1961-1965
}

\section{Zentrale Institutionen und Instrumente zur Steuerung des Militärs}

Der SED war es bereits bis zum Mauerbau gelungen, die organisatorische Ausformung der zentralen Institutionen und Instrumente zur Kontrolle und Steuerung der militärischen Diskussions- und Entscheidungsprozesse weitgehend zu vollenden. In diesem Bereich ihres Interventionssystems sah sich die Partei nach 1961 daher nur noch dazu veranlaßt, einige Modifikationen vorzunehmen, um die vorhandenen Strukturen den veränderten militärpolitischen Zielsetzungen und Rahmenbedingungen anzupassen.

So änderte etwa das Politbüro durch einen Beschluß vom 12. November 1963 die Zusammensetzung des Nationalen Verteidigungsrates. Sowohl der Chef des Hauptstabes der NVA, zu diesem Zeitpunkt Generalleutnant Siegfried Riedel, als auch der Chef der Politischen Hauptverwaltung, Admiral Waldemar Verner, wurden nunmehr voll stimmberechtigte Mitglieder dieses Gremiums ${ }^{1}$. Bis dahin hatten sie nur mit beratender Stimme teilgenommen ${ }^{2}$. Diese Veränderung resultierte vermutlich vor allem aus dem Umstand, daß sich der Nationale Verteidigungsrat nach dem Mauerbau im Gegensatz zur Sicherheitskommission in den fünfziger Jahren vermehrt mit militärischen Fragen der äußeren Sicherheit befaßte ${ }^{3}$ und man diese beiden Stellvertreter des Ministers deshalb stärker in die politischen Entscheidungsprozesse einbeziehen wollte.

Ferner gab es im neuen Statut vom 14. Dezember 1963 einen Zusatz hinsichtlich der Arbeitsweise des Nationalen Verteidigungsrates. Ausdrücklich wurde jetzt der Beschlußfassungsmodus bestimmt: Prinzipielle Beschlüsse des Gremiums waren "mit Stimmenmehrheit" zu fassen". So war es zumindest formal möglich, Ulbricht als Vorsitzenden des Nationalen Verteidigungsrates zu überstimmen. Es könnte daher an diesem neuen Passus gelegen haben, daß Ulbricht es als notwendig erachtete, im Hinblick auf den Einsatz der Streitkräfte über die Festlegungen des Statuts hinaus sich selbst als Vorsitzendem beziehungsweise Honecker in seiner Funktion als Sekretär eine privilegierte Entscheidungsbefugnis einzuräumen. Dementsprechend

1 Zusammensetzung des Nationalen Verteidigungsrates der DDR. Protokoll Nr. 40/63 der Sitzung des Politbüros des ZK der SED, 12. November 1963, SAPMO-BArch, DY 30/J IV 2/2/905, Bl. 98.

2 Vgl. S. $79 \mathrm{ff}$.

3 Vgl. Wagner, Der Nationale Verteidigungsrat der DDR, S. $174 \mathrm{ff}, 181 \mathrm{ff}$.

4 Statut des Nationalen Verteidigungsrates der DDR. Protokoll der 17. Sitzung des Nationalen Verteidigungsrates der DDR, 14. Dezember 1963, BA-MA, DVW 1/39474, Bl. 14. 
befahl Ulbricht 1965: „Der Einsatz der Nationalen Volksarmee zur Lösung von Gefechtsaufgaben im Bestand der Vereinten Streitkräfte der Staaten des Warschauer Vertrages sowie der Einsatz zur Lösung anderer Gefechtsaufgaben, wie zum Beispiel Zerschlagung von gegnerischen Gruppierungen[,] die den verdeckten Kampf führen, Übergang zur gefechtsmäßigen Sicherung der Staatsgrenze und ähnliches, bedarf meiner Bestätigung bzw. der des Sekretärs des Nationalen Verteidigungsrates." 5 Grundsätzlich allerdings dürften Mehrheitsbeschlüsse gegen die erklärte Auffassung Ulbrichts, der zugleich auch 1. Sekretär des ZK und Vorsitzender des Staatsrates war6, unrealistisch gewesen sein, hätten sie doch automatisch Ulbrichts Machtposition innerhalb der Parteispitze in Frage gestellt.

Eine weitere organisatorische Neuerung war die Umwandlung der Politischen Verwaltung des Ministeriums für Nationale Verteidigung in die „Politische Hauptverwaltung der Nationalen Volksarmee" zum 1. Oktober 1961. Parallel dazu wurden die Politabteilungen der Kommandos der Teilstreitkräfte und der Militärbezirke zu Politischen Verwaltungen aufgewertet ${ }^{7}$. In seinem Befehl begründete Hoffmann diese Maßnahme vor allem mit der Absicht einer ,weiteren Verbesserung der politischen Führungs- und Erziehungsarbeit" 8 . Insofern fügte sich die Schaffung der Politischen Hauptverwaltung in die allgemeine Zielsetzung der SED nach 1961 ein, die Politorgane vor dem Hintergrund der Konsolidierung der NVA außer zur Kontrolle der militärischen Truppenführung in Zukunft noch stärker zur Mobilisierung und Erziehung der Armeeangehörigen zu verwenden'. Im Zusammenhang mit dieser Intention wurden die Aufgaben der Politischen Hauptverwaltung durch die neue „Instruktion für die Parteiorganisationen der SED in der NVA und für die Politorgane der NVA“, die das Politbüro am 5. November 1963 bestätigte, noch einmal erweitert. So war das „leitende Organ für die politische Arbeit“ jetzt unter anderem auch dafür zuständig, die Lehrpläne für das "gesellschaftswissenschaftliche Studium" an der Militärakademie und den Schulen der NVA zu erstellen sowie die „wissenschaftliche Forschungsarbeit" der Lehrstühle für Gesellschaftswissenschaft zu kontrollieren ${ }^{10}$. Es ist daher nicht verwunderlich, daß die Bildung der Politischen Hauptverwaltung mit einer deutlichen quantitativen Steigerung des Politoffiziersbestandes innerhalb eines Jahres von 2790 Offizieren Anfang 1961 auf 3538 im Januar 1962 einherging ${ }^{11}$. Eine weiterer Grund für diese Umstrukturierungs-

${ }^{5}$ Festlegung der Entscheidungsbefugnisse über den Einsatz der NVA. Befehl Nr. 3/65 des Vorsitzenden des Nationalen Verteidigungsrates der DDR, 1965, BA-MA, VA-01/40335, Bl. 167.

6 Zusammensetzung des Nationalen Verteidigungsrates der DDR. Protokoll Nr. 40/63 der Sitzung des Politbüros des ZK der SED, 12. November 1963, SAPMO-BArch, DY 30/J IV 2/2/905, Bl. 98.

7 Einführung neuer Bezeichnungen für die Politische Verwaltung des Ministeriums für Nationale Verteidigung und die Politabteilungen der Kommandos der Teile und Militärbezirke. Befehl Nr. 82/61 des Ministers für Nationale Verteidigung, 18. Oktober 1961, BA-MA, VA-01/5906, Bl. $67 \mathrm{f}$.

8 Ebenda, Bl. 67.

9 Vgl. Instruktion für die Parteiorganisationen der SED in der NVA und für die Politorgane der NVA. Protokoll Nr. 39/63 der Sitzung des Politbüros des ZK der SED, 5. November 1963, SAPMO-BArch, DY 30/J IV 2/2/904, Bl. $154 \mathrm{ff}$.

10 Ebenda, Bl. 160 f.

11 Stärkenachweisbuch - Gesamtbestand an Offizieren der NVA, Januar 1957-Juni 1967, BA-MA, VA-01/32458, Bl. $20 \mathrm{ff}$. 
maßnahme dürfte gewesen sein, daß die Politorgane der NVA jetzt „im wesentlichen der Struktur der im Warschauer Vertrag vereinten sozialistischen Armeen“ angeglichen werden sollten ${ }^{12}$.

Im Rahmen der neuen Instruktion von 1963 wurde noch eine weitere organisatorische Veränderung verwirklicht. Die Politische Hauptverwaltung erhielt ein eigenes „Sekretariat", in dem alle grundsätzlichen Fragen der Parteiarbeit „beraten und beschlossen“ werden sollten. Diese Beschlüsse wurden im Anschluß durch Direktiven und Anordnungen des Chefs der Politischen Hauptverwaltung implementiert $^{13}$. Da das Sekretariat laut Instruktion zur „Festigung der Kollektivität bei der Beratung" geschaffen wurde ${ }^{14}$, drängt sich die Vermutung auf, daß es das Ziel der Parteiführung war, in Zukunft unter anderem anhand dieses Gremiums eine wirksame Kontrolle der Leitung der Politischen Hauptverwaltung sicherzustellen.

Auch im Hinblick auf das Kadernomenklatursystem, mit dessen Hilfe die SEDFührung weiterhin die Besetzung aller militärischen Leitungspositionen steuerte, gab es eine grundsätzliche organisatorische Änderung. So läßt das neue Verzeichnis der Nomenklaturdienststellungen aus dem Bereich der Streitkräfte die tendenzielle Absicht erkennen, die obersten Parteigremien in dieser Frage zu entlasten und ihre Entscheidungskompetenz auf die Besetzung der wichtigsten Dienststellungen zu beschränken. Ein Teil der Nomenklaturkader, die bisher in den Nomenklaturen der führenden Parteigremien enthalten waren, wurde daher in die Nomenklaturen des staatlichen Bereichs verlagert, so daß über die Besetzung zentraler Leitungspositionen der NVA nunmehr zunehmend in der Leitung des Ministeriums für Nationale Verteidigung entschieden wurde. Offenbar schätzte die SED ihr Regime nach dem Mauerbau auch in den Streitkräften als so gefestigt ein, daß sie im Zusammenhang mit dem Kadernomenklatursystem eine gewisse funktionale Differenzierung zuließ. Zudem war es der Partei in den Jahren zuvor gelungen, alle militärischen Führungspositionen mit hohen SED-Funktionären zu besetzen, die über ihre jeweiligen Nomenklaturen ohnehin die Besetzung der herausgehobenen Dienststellungen in den nachgeordneten Ebenen und Bereichen kontrollierten.

Gemäß der neuen Nomenklatur, die am 1. Juli 1962 in Kraft trat, entschied das Politbüro jetzt über die Besetzung der Dienststellungen des Ministers für Nationale Verteidigung sowie des Stellvertreters des Ministers und Chefs der Politischen Hauptverwaltung. Letztere war bis dahin in der Nomenklatur des Nationalen Verteidigungsrates geführt worden, erfuhr aber mit der Umbildung des leitenden Parteiorgans in der NVA auch hier eine Aufwertung. Ferner entschied das Politbüro über alle Ernennungen und Beförderungen von Generalen und Admiralen ${ }^{15}$. Die Dienststellungen der übrigen Stellvertreter des Ministers für Nationale Verteidi-

12 Prinzipien für die Struktur der Politorgane, Partei- und FDJ-Organe in der NVA für 1962 und vorgesehene Veränderungen der Struktur der Politorgane, des Partei- und FDJ-Aufbaus in der NVA. Protokoll der Sitzung der Leitung des Ministeriums für Nationale Verteidigung, 28. Juli 1961, BA-MA, AZN/28079, Bl. 12.

13 Instruktion für die Parteiorganisationen der SED in der NVA und für die Politorgane der NVA. Protokoll Nr. 39/63 der Sitzung des Politbüros des ZK der SED, 5. November 1963, SAPMO-BArch, DY 30/J IV 2/2/904, Bl. 160.

14 Ebenda, Bl. 153.

15 Verzeichnis der Dienststellungen der Nomenklatur, 12. Juni 1962, BA-MA, VA-01/23771, Bl. 2. 
gung, wie des Chefs des Hauptstabes oder des Chefs Rückwärtige Dienste, wurden jetzt in der Nomenklatur des Ministerrates geführt. Der Ministerrat bestätigte formal auch die Entscheidungen über die Ernennungen und Beförderungen von Generalen und Admiralen, die zuvor durch das Politbüro gefällt worden waren. Die Beschlußfassung des Ministerrates über Nomenklaturkader der Streitkräfte erfolgte ohnehin erst nach „vorheriger Zustimmung durch den Nationalen Verteidigungsrat", so daß die SED-Führung letztlich auch hier immer den bestimmenden Einfluß ausübte ${ }^{16}$. In der Nomenklatur des ZK-Sekretariats wurde nunmehr die Dienststellung des Chefs der Verwaltung Kader geführt, was insofern konsequent war, als sich das Sekretariat zu einem großen Teil mit Kaderfragen befaßte ${ }^{17}$.

Die Nomenklatur des Nationalen Verteidigungsrates war vor allem um die Stellvertreter des Ministers für Nationale Verteidigung verkleinert worden. Sie enthielt jetzt noch die Dienststellungen des Chefs der Verwaltung Ausbildung und der Chefs Artillerie, Nachrichten, Pionierwesen und Aufklärung. Ferner umfaßte die Nomenklatur die Chefs der Operativen sowie der 12. und 15. Verwaltung, die Stellvertreter des Chefs des Hauptstabes für Organisation und des Chefs der Politischen Hauptverwaltung sowie die Leiter der 14. Abteilung, der Auslandsabteilung und der Hauptabteilung Militärjustiz im Ministerium der Justiz sowie den Militäroberstaatsanwalt. Zudem wurden die Dienststellungen der Militärattachés in der UdSSR, der VR China, der VR Polen und der ČSSR durch den Nationalen Verteidigungsrat besetzt, ebenso wie die der Chefs der Militärbezirke und ihrer Stellvertreter, der Chefs der Volksmarine und der Grenztruppen und ihrer Stellvertreter sowie der Stellvertreter des Chefs der Luftstreitkräfte/Luftverteidigung. Weiterhin enthielt die Nomenklatur alle Divisionskommandeure der Landstreitkräfte und der Luftstreitkräfte/Luftverteidigung sowie die Flottillenchefs der Volksmarine und die Kommandeure der Grenzbrigaden. Sie endete mit der Dienststellung des Kommandeurs der Militärakademie „Friedrich Engels“ und seines 1. Stellvertreters ${ }^{18}$.

Im Hinblick auf die Nomenklatur der ZK-Abteilung für Sicherheitsfragen gab es 1962 eine Reihe von Änderungen. Sie wurde deutlich von rund 335 Dienststellungen Anfang $1961^{19}$ auf 242 Mitte $1962^{20}$ reduziert $^{21}$. Ein Teil der Dienststellungen,

16 Ebenda.

17 Ebenda.

18 Ebenda, Bl. $2 \mathrm{f}$.

19 Vgl. S. $88 \mathrm{ff}$.

20 Verzeichnis der Dienststellungen der Nomenklatur, 12. Juni 1962, BA-MA, VA-01/23771, Bl. 1.

21 Die Nomenklatur umfaßte im wesentlichen die Dienststellungen der Chefs der Verwaltungen Organisation und Planung, Werbung und Auffüllung sowie Planung, ebenso gehörte dazu die Dienststellung des Chefs Kraftfahrzeug-Wesen. Zudem beinhaltete sie den Stellvertreter des Chefs des Hauptstabes für allgemeine Fragen und die Stellvertreter des Chefs der Verwaltung Kader sowie den 1. Sekretär der Kreisleitung des Ministeriums für Nationale Verteidigung und den 1. Sekretär der Parteileitung der Militärakademie. Auch die Militärattachés in Ungarn, Rumänien, Bulgarien, Korea und Vietnam, der 1. Stellvertreter des Militäroberstaatsanwalts sowie die Kommandeure der Schulen der Teilstreitkräfte wurden in der Nomenklatur der ZK-Abteilung für Sicherheitsfragen geführt ebenso wie die Stellvertreter der Leiter der Politischen Verwaltungen der Militärbezirke und Teilstreitkräfte. Sie enthielt die Dienststellungen der Chefs Artillerie und Luftabwehr sowie die Leiter der Kaderabteilungen der Kommandos der Militärbezirke und der Chefs der Wehrbezirkskommandos. Außerdem war die ZK-Abteilung für die Besetzung der Dienststellungen der 1. Stellvertre- 
die bisher hier verzeichnet waren, überführte man in die Nomenklaturen der Leitung des Ministeriums für Nationale Verteidigung. So gingen etwa die Dienststellungen der Parteisekretäre der Kommandos der Militärbezirke und Teilstreitkräfte sowie der Regimentsparteisekretäre in die „Kontrollnomenklatur"22 der Leitung des Ministeriums ein ${ }^{23}$. Letztlich überwachte die ZK-Abteilung für Sicherheitsfragen jedoch weiterhin die Besetzung dieser Positionen, wenn sie darüber selbst auch nicht mehr entschied. Denn die Leitung des Ministeriums mußte sich alle ihre Entscheidungen über Nomenklaturkader nach dem Beschluß der Kadervorlage durch die ZK-Abteilung für Sicherheitsfragen bestätigen lassen ${ }^{24}$.

\section{Der Parteiapparat}

Trotz der Tatsache, daß dem Parteiapparat der SED in der NVA in der Phase der Konsolidierung und Professionalisierung der Streitkräfte ab Mitte 1961 im Hinblick auf die militärische Führungstätigkeit eine mehr kontrollierende als steuernde Funktion zukam, führte dies zu keiner Reduzierung seines Umfanges. Der Parteiapparat erfuhr nach dem Mauerbau sogar noch eine quantitative Erweiterung. Neben einer deutlichen Zunahme der Zahl der Politoffiziere ${ }^{25}$ erhöhte sich nicht zuletzt auch die Zahl der Parteimitglieder und -kandidaten in der NVA von $37762 \mathrm{Ar}$ meeangehörigen 1958 auf 57518 im Jahr $1963^{26}$.

Die SED-Führung vollzog jedoch auch einige organisatorische Modifikationen innerhalb des Parteiapparates ${ }^{27}$. In bezug auf die Parteiorganisationen beschloß das Sekretariat des ZK im März 1962 eine Änderung der noch immer gültigen „Instruktion für die Arbeit der Parteiorganisationen der SED in der NVA "von 1958: Parteigrundorganisationen waren jetzt nicht mehr auf der Ebene der Regimenter, sondern

ter, Politstellvertreter und Stabschefs der Divisionen der Landstreitkräfte sowie der Stellvertreter des Kommandeurs der Luftverteidigungsdivision verantwortlich, ferner für die Besetzung der Dienststellungen der Politstellvertreter und Stabschefs der Flottillen sowie der Chefs der Brigaden der Volksmarine. Schließlich enthielt die Nomenklatur die Funktionen der Politstellvertreter und Stabschefs der Grenzbrigaden der Grenztruppen ebenso wie der Regiments- und Geschwaderkommandeure der NVA. Außerdem hatte die ZK-Abteilung für Sicherheitsfragen weiterhin über alle Beförderungen zum Oberst und alle Delegierungen zu den Militärakademien der UdSSR zu entscheiden. Verzeichnis der Dienststellungen der Nomenklatur, 12. Juni 1962, BA-MA, VA-01/23771, Bl. $16 \mathrm{ff}$.

22 Kontrollnomenklaturen enthielten Dienststellungen, die aufgrund ihrer nachgeordneten $\mathrm{Be}-$ deutung nicht in der eigentlichen Nomenklatur eines Bereiches geführt wurden. Der jeweilige Nomenklaturvorgesetzte konnte sich anhand der Kontrollnomenklaturen jedoch jederzeit einen Eindruck von der personellen Besetzung dieser Dienststellungen verschaffen. Dies war insofern von Bedeutung, als diese Kader in der Regel als potentieller Nachwuchs für die Besetzung von Dienststellungen der Kadernomenklaturen galten. Vgl. Wagner, Das Nomenklatursystem, S. 27.

23 Verzeichnis der Dienststellungen der Nomenklatur, 12. Juni 1962, BA-MA, VA-01/23771, Bl. $9 \mathrm{ff}$.

24 Ebenda, Bl. 16.

25 Vgl. Kap. VIII.1. dieser Studie. Zu einem gewissen Teil war diese Zunahme jedoch auch auf die am 15. September 1961 vollzogene Eingliederung der Grenztruppen in die NVA zurückzuführen.

26 Wenzke, Die Nationale Volksarmee, S. 438.

27 Vgl. Herspring, East German Civil-Military Relations, S. $101 \mathrm{ff}$. 
bereits auf der der Bataillone und selbständigen Kompanien sowie Einheiten vergleichbarer Größe zu bilden ${ }^{28}$. Auf der Ebene der Züge und Kompanien gliederten sich diese Grundorganisationen in Parteigruppen ${ }^{29}$. Die Parteiorganisationen der Regimenter, die mit einer zivilen Betriebsparteiorganisation vergleichbar waren, setzten sich wiederum aus den Grundorganisationen der Bataillone zusammen ${ }^{30}$. Nach Auffassung der Leitung des Ministeriums für Nationale Verteidigung wurde diese Änderung erforderlich, weil die „,bisher bestehenden Parteigrundorganisationen bei den Regimentern und Gleichgestellten es nicht ermöglichten, entsprechend dem Statut der Partei ein Parteileben der Grundorganisationen zu entwickeln"31. Vermutlich war es angesichts der steigenden Mitgliederzahlen nötig geworden, die operative Parteiarbeit auf die darunterliegende Ebene zu verlagern ${ }^{32}$.

Darüber hinaus gab es im Zusammenhang mit der Umwandlung der Politischen Verwaltung des Ministeriums für Nationale Verteidigung in die Politische Hauptverwaltung der NVA im Oktober 1961 strukturelle Veränderungen auch in den nachgeordneten Politorganen: Zunächst wurden in den Kompanien und Batterien der Bataillone und Abteilungen sowie in den gleichgestellten Einheiten der übrigen Teilstreitkräfte „keine Politstellvertreter mehr eingesetzt“33. Nach Ansicht der SED war dies möglich, weil sich das „allgemeine Niveau“ - sowohl die politische Zuverlässigkeit als auch die Führungsqualitäten - der Offiziere verbessert hatte. Damit glaubte man, gleichzeitig auch die Verwirklichung der „Einheit von politischer und militärischer Führung" weiter voranzutreiben ${ }^{34}$. Selbständige Kompanien und Batterien behielten jedoch ihre Politstellvertreter, da diese Einheiten nicht Teile eines Bataillons waren und anderenfalls keiner unmittelbaren Kontrolle durch ein Politorgan unterstanden hätten ${ }^{35}$.

Parallel zu ihren erweiterten Aufgaben, die detailliert Eingang in die neue „Instruktion für die Parteiorganisationen der SED in der NVA und für die Politorgane der NVA“ von 1963 fanden ${ }^{36}$, erfolgte von der Bataillonsebene an aufwärts auch

28 Änderung der bestätigten „Instruktion für die Arbeit der Parteiorganisationen der SED in der NVA“. Protokoll Nr. 14/62 der Sitzung des Sekretariats des ZK der SED, 21. März 1962, SAPMO-BArch, DY 30/J IV 2/3/797, Bl. 5, 11.

29 Beratung und Beschlußfassung von Strukturfragen der Politorgane sowie des Partei- und FDJ-Aufbaus in der NVA. Protokoll Nr. 9/61 der Sitzung der Leitung der Politischen Verwaltung, 22. Juni 1961, BA-MA, VA-P-01/027, Bl. 167.

30 Prinzipien für die Struktur der Politorgane, Partei- und FDJ-Organe in der NVA für 1962 und vorgesehene Veränderungen der Struktur der Politorgane, des Partei- und FDJ-Aufbaus in der NVA. Protokoll der Sitzung der Leitung des Ministeriums für Nationale Verteidigung, 28. Juli 1961, BA-MA, AZN/28079, Bl. 16.

31 Ebenda, Bl. 15.

32 Tatsächlich handelte es sich bei dieser Änderung um keine wirkliche Neuerung. Vielmehr führte man Organisationsstrukturen wieder ein, die schon einmal im Rahmen der Instruktion von 1957 existiert hatten. Vgl. S. $99 \mathrm{ff}$.

33 Veränderungen in den Politorganen der NVA. Verner an Borning, 14. Oktober 1961, SAPMO-BArch, DY 30/IV 2/12/9, Bl. 203.

34 Prinzipien für die Struktur der Politorgane, Partei- und FDJ-Organe in der NVA für 1962 und vorgesehene Veränderungen der Struktur der Politorgane, des Partei- und FDJ-Aufbaus in der NVA. Protokoll der Sitzung der Leitung des Ministeriums für Nationale Verteidigung, 28. Juli 1961, BA-MA, AZN/28079, Bl. 11.

35 Veränderungen in den Politorganen der NVA. Verner an Borning, 14. Oktober 1961, SAPMO-BArch, DY 30/IV 2/12/9, Bl. 203.

36 Instruktion für die Parteiorganisationen der SED in der NVA und für die Politorgane der 
eine organisatorische Spezifizierung der Politorgane. So wurde den Politoffizieren der Bataillone, der Abteilungen der Regimenter und der selbständigen Bataillone der Divisionen ein Offizier für Agitation und Propaganda unterstellt. In den selbständigen Bataillonen der Militärbezirke und Teilstreitkräfte erhielt das Politorgan zur Verstärkung einen Offizier für Organisationsfragen und politische Massenarbeit ${ }^{37}$. Ferner bekam der Politapparat auf Regimentsebene die Bezeichnung „Politgruppe“. Die Politabteilungen der Divisionen untergliederten sich neuerdings in Unterabteilungen für Organisation und Instruktion sowie für Agitation und Propaganda. Die Politabteilungen der Militärbezirke und Teilstreitkräfte ihrerseits wurden in Politische Verwaltungen umbenannt. Sie waren ebenfalls in Abteilungen für Organisation und Instruktion sowie für Agitation und Propaganda unterteilt. Diese Struktur gab es auch auf der Ebene der Politischen Hauptverwaltung, wobei es sich hier um eigene Verwaltungen handelte ${ }^{38}$.

Darüber hinaus war bereits im April 1961 nach Zustimmung des Sekretariats des ZK eine Kreisleitung der SED im Ministerium für Nationale Verteidigung gebildet worden ${ }^{39}$. Während diese in erster Linie die politische Arbeit in den Abteilungen und Verwaltungen des Ministeriums organisierte, übernahm die Politabteilung des Ministeriums die parteipolitischen Aufgaben in den Truppenteilen und Einrichtungen, die dem Ministerium direkt unterstellt waren ${ }^{40}$. Eine weitere SED-Kreisleitung wurde 1963 auch in der Militärakademie „Friedrich Engels" geschaffen ${ }^{41}$.

Die entscheidende Modifikation, die die SED-Führung in der Phase zwischen 1961 und 1965 im Parteiapparat vornahm, betraf jedoch die Politstellvertreter und Parteisekretäre in den Einheiten. Dabei ging es zum einen um deren Verhältnis zueinander und zum anderen um die Kompetenzen, die sie besaßen, um Einfluß auf die spezifisch militärischen Diskussions- und Entscheidungsprozesse zu nehmen. Diesbezüglich galten nach dem 13. August 1961 formal zunächst auch weiterhin die Vorschriften, die bereits 1958/1959 fixiert worden waren. Demzufolge verfügten die Parteisekretäre in der Truppe über den größten Einfluß ${ }^{42}$.

NVA. Protokoll Nr. 39/63 der Sitzung des Politbüros des ZK der SED, 5. November 1963, SAPMO-BArch, DY 30/J IV 2/2/904, Bl. $154 \mathrm{ff}$.

37 Veränderungen in den Politorganen der NVA. Verner an Borning, 14. Oktober 1961, SAPMO-BArch, DY 30/IV 2/12/9, Bl. 204.

38 Prinzipien für die Struktur der Politorgane, Partei- und FDJ-Organe in der NVA für 1962 und vorgesehene Veränderungen der Struktur der Politorgane, des Partei- und FDJ-Aufbaus in der NVA. Protokoll der Sitzung der Leitung des Ministeriums für Nationale Verteidigung, 28. Juli 1961, BA-MA, AZN/28079, Bl. 12.

39 Bildung einer Kreisleitung im Bereich des Ministeriums für Nationale Verteidigung. Protokoll Nr. 17/61 der Sitzung des Sekretariats des ZK der SED, 19. April 1961, SAPMO-BArch, DY 30/J IV 2/3/734, Bl. 2.

40 Prinzipien für die Struktur der Politorgane, Partei- und FDJ-Organe in der NVA für 1962 und vorgesehene Veränderungen der Struktur der Politorgane, des Partei- und FDJ-Aufbaus in der NVA. Protokoll der Sitzung der Leitung des Ministeriums für Nationale Verteidigung, 28. Juli 1961, BA-MA, AZN/28079, Bl. 16.

41 Instruktion für die Parteiorganisationen der SED in der NVA und für die Politorgane der NVA. Protokoll Nr. 39/63 der Sitzung des Politbüros des ZK der SED, 5. November 1963, SAPMO-BArch, DY 30/J IV 2/2/904, Bl. 153.

42 Vgl. S. $99 \mathrm{ff}$. 
Im Zusammenhang mit der Abkehr von der chinesischen Militärkonzeption 1960/1961 $1^{43}$ und der zunehmenden Professionalisierung der NVA ${ }^{44}$ scheint es dann entgegen den noch gültigen Bestimmungen in der Praxis zu einer zunehmenden Aufgabe dieses Organisationsschemas gekommen zu sein. Ende 1962 hieß es dementsprechend: „In den Fragen der Kaderauslese, Förderung und Entwicklung der bewaffneten Organe wurde doch den Parteiorganisationen durch den Beschluß des Politbüros vom 14.01. 1958 größter Einfluß eingeräumt. Tatsache ist aber, daß eine Vielzahl von Einheiten, Truppenteilen und Dienststellen mit diesem Dokument in den letzten zwei Jahren wenig bzw. überhaupt nicht mehr gearbeitet haben. “45 Die schleichende Revision der exponierten Rolle der Parteisekretäre seit 1961 wurde schließlich im Rahmen der neuen Instruktion vom 5. November 1963 von der SED auch formal vollzogen.

Ein entscheidender Grund für diese Entwicklung war zum einen, daß sich die Einflußnahme auf die militärischen Entscheidungsprozesse durch die Parteisekretäre vor dem Hintergrund der von der SED-Führung angestrebten substantiellen Verbesserung der Leistungsfähigkeit der NVA im Zuge ihrer Integration in die Erste Strategische Staffel zunehmend als ineffizient herausstellte. Die Kritik an der Führungstätigkeit der Kommandeure und Offiziere in den Parteiorganisationen mochte Ende der fünfziger Jahre dazu geeignet gewesen sein, den Widerstand des Offizierskorps gegen die Verwirklichung der "führenden Rolle“ der SED in der Armee zu brechen, einer Steigerung der Einsatzbereitschaft stand sie jedoch nachhaltig im Wege. Da diese Praxis nicht zuletzt die Autorität der Vorgesetzten untergrub und die militärische Entscheidungsfindung empfindlich störte, wurde nicht parteikonformes Verhalten leitender Offiziere nach dem Mauerbau auf Parteiversammlungen etwa durch Parteistrafen oder die Anwendung des Prinzips der Kritik und Selbstkritik immer seltener sanktioniert ${ }^{46}$.

Insofern wurde beinahe zwangsläufig die Stellung der Politstellvertreter wieder gestärkt. Da sie den Stäben ihrer Einheiten regulär angehörten, konnten sie dort in bezug auf die militärischen Diskussions- und Entscheidungsprozesse jederzeit die Position der Partei vertreten. Auf diese Weise ließ sich die politische Kontrolle reibungsloser und damit effizienter ausüben. Dabei dürfte es sich als hilfreich erwiesen haben,"daß die Politstellvertreter mittlerweile über eine immer bessere militärische Qualifikation verfügten, was im Hinblick auf die immer komplexeren Gefechtsaufgaben, die die NVA zu erfüllen hatte, von entscheidender Bedeutung war. Diese Veränderungen scheinen nicht zuletzt von leitenden Vertretern der Politorgane selbst gefordert und vorangetrieben worden zu sein. Diese suchten nach dem 13. August 1961 offenbar umgehend, den Ende der fünfziger Jahre erfolgten Machtverlust zu kompensieren ${ }^{47}$. Hierauf deutet beispielsweise die Äußerung eines Mitarbeiters der Verwaltung für Agitation und Propaganda der Politischen Hauptverwal-

43 Vgl. Kap. V.3. dieser Studie.

44 Vgl. Kap. VII.3. dieser Studie.

45 Die Ergebnisse im Ausbildungsjahr 1962, SAPMO-BArch, DY 30/IV 2/12/38, Bl. 219.

46 Vgl. Kap. VII.3. dieser Studie.

47 Diese Absicht läßt der erste Entwurf für die Überarbeitung der „Instruktion für die Arbeit der Parteiorganisationen der SED in der NVA“ und der „Bestimmungen für die Arbeit der Politorgane der NVA“ erkennen, den die Politische Hauptverwaltung Ende 1961 erarbeitet hatte. Information, 17. November 1961, SAPMO-BArch, DY 30/IV 2/12/15, Bl. $143 \mathrm{ff}$. 
tung hin, der Ende des Jahres sagte: „Ich denke, die Parteisekretäre sollen jetzt eine untergeordnete Rolle spielen." 48

Der SED kam dabei zugute, daß sich das Verhältnis zwischen der Partei und dem Offizierskorps als Folge des Mauerbaus zunehmend normalisierte und sich die Mehrheit der Offiziere mit der umfassenden Präsenz der SED in den Streitkräften arrangierte.

Als das ZK der KPdSU am 17. Januar 1963 die neue „Instruktion für die Parteiorganisation der KPdSU in der Sowjetarmee und Seekriegsflotte" und die neuen „Bestimmungen über die Politorgane der Sowjetflotte und Seekriegsflotte“ bestätigte $^{49}$, wurde deutlich sichtbar, daß die Stellung der Politstellvertreter in der NVA im Vergleich zu den sowjetischen Streitkräften grundsätzlich unterschiedlich geregelt war. Offenbar hielt es die SED-Führung nunmehr für geboten, eine formale Angleichung zu vollziehen. Bei der Auswertung der neuen sowjetischen Vorschriften arbeitete die ZK-Abteilung für Sicherheitsfragen die Abweichungen klar heraus. So hieß es, die Politstellvertreter beziehungsweise die Politorgane in der NVA hätten gemäß der noch gültigen Instruktion von 1958 die Arbeit der Parteiorganisationen allein anzuleiten und die Tätigkeit der Parteileitungen und Parteiaktive zu „unterstützen“. Im Gegensatz dazu würden die Politstellvertreter in den sowjetischen Streitkräften für die Parteiarbeit, die sie zusammen mit den Parteileitungen und den Parteisekretären organisierten, jedoch die „unmittelbare Verantwortung“ tragen ${ }^{50}$. Bei der Überarbeitung der Instruktion und der Bestimmungen von 1958 wurden diese prinzipiellen Unterschiede daher beseitigt. Indem die Parteisekretäre ihre herausgehobene Stellung verloren, wurden zugleich auch die letzten Überreste der chinesischen Militärkonzeption in der NVA getilgt. Deren Ursprung dürfte zu diesem Zeitpunkt jedoch außer den politisch und militärisch Verantwortlichen den meisten Armeeangehörigen nicht zuletzt wegen der Tabuisierung dieses Themas kaum mehr bekannt gewesen sein.

Durch die neue „Instruktion für die Parteiorganisationen der SED in der NVA und für die Politorgane der NVA “ von 1963 wurde der Funktionswechsel der Politstellvertreter und Parteisekretäre formal implementiert: Neben der Zuständigkeit für politische Fragen hatten die Politstellvertreter jetzt ausdrücklich auch die Aufgabe, „sachkundig auf die Erfüllung aller Aufgaben der Gefechtsausbildung Einfluß zu nehmen“ und "rechtzeitig Maßnahmen zur Beseitigung von Mängeln zu ergreifen, die die Erhöhung der Gefechtsbereitschaft" behinderten. Zudem sollten sie die "Wirksamkeit" der Ausbildung regelmäßig ,analysieren“51. Die neue Instruktion ermächtigte die Politstellvertreter dazu, diese Aufgaben unmittelbar im Rahmen der militärischen Truppenführung zu erfüllen: zum einen, weil die Vorschrift einmal mehr die „Einzelleitung auf parteilicher Grundlage“ postulierte, was bedeutete, daß

48 Ebenda, Bl. 141.

49 Information über die Berücksichtigung der Parteiinstruktion und der Bestimmungen über die Politorgane der Sowjetarmee bei der Überarbeitung der Instruktion für die Parteiorganisationen und Politorgane in der NVA, 5. Dezember 1963, SAPMO-BArch, DY 30/IV A2/12/34, o. Pag.

50 Ebenda.

51 Instruktion für die Parteiorganisationen der SED in der NVA und für die Politorgane der NVA. Protokoll Nr. 39/63 der Sitzung des Politbüros des ZK der SED, 5. November 1963, SAPMO-BArch, DY 30/J IV 2/2/904, Bl. $154 \mathrm{ff}$. 
das Prinzip der kollektiven Beratung aller Entscheidungen auch weiterhin galt ${ }^{52}$. Die Politstellvertreter waren auf diese Weise immer an den Entscheidungen ihrer Kommandeure beteiligt.

Zum anderen verfügten sie innerhalb der militärischen Leitungen über eine große Eigenständigkeit, die es ihnen ermöglichte, eine wirkungsvolle Kontrolle der militärischen Truppenführung auszuüben. Denn sie waren den Kommandeuren allein als „Stellvertreter für politische Arbeit" unterstellt ${ }^{53}$. „Als leitende Parteifunktionäre“ unterstanden die Politstellvertreter aber ausschließlich dem „Leiter des nächsthöheren Politorgans" 54 . Dadurch konnten sie unabhängig vom militärischen Dienstweg der in der Instruktion enthaltenen Verpflichtung nachkommen, „ernsthafte Unstimmigkeiten in der Befehlsgebung“, die mit den Kommandeuren nicht zu „klären“ waren, oder Verletzungen von Parteibeschlüssen und Gesetzen, „sofort" dem übergeordneten Parteiorgan, wenn nötig sogar dem ZK selbst zu melden ${ }^{55}$. Diesbezüglich diente es offenkundig der Wahrung der militärischen Effizienz, daß diese Bestimmung eine Einbeziehung der Parteileitungen und Parteisekretäre in derartigen Situationen explizit nicht mehr vorsah. Im übrigen war die dienstrechtliche Stellung der Politstellvertreter besonders gegenüber den Kommandeuren bereits dadurch gestärkt worden, daß der Politstellvertreter in Abänderung der Innendienstvorschrift von 1959 nun wieder "direkter Vorgesetzter aller Angehörigen“" seiner Einheit war ${ }^{56}$.

Die Parteisekretäre und Parteiorganisationen verfügten jetzt über keinen entscheidenden Einfluß auf die spezifisch militärischen Entscheidungsprozesse mehr. Die Instruktion gab den Parteisekretären der Einheiten zwar noch das „Recht, an den Beratungen der Kommandeure mit ihren Stellvertretern und an der Attestierung der Offiziere teilzunehmen " 57 , eine dezidierte Verpflichtung der Kommandeure, die Parteisekretäre vor allen wichtigen militärischen Entscheidungen zu hören, bestand aber nicht mehr. Im Hinblick auf Status und Kompetenzen rangierten die Parteisekretäre jetzt immer hinter den Politstellvertretern. So hatten letztere beispielsweise auf die Kaderarbeit maßgeblichen Einfluß zu nehmen, jedoch nur „unter Berücksichtigung der Vorschläge der Parteiorganisationen" 58 .

Ein Parteisekretär war jetzt in erster Linie für die Leitung der laufenden Arbeit der Parteiorganisation zuständig. Sogar den Entwurf des Arbeitsplanes der jeweiligen Parteileitung erstellte er nicht mehr selbständig, sondern „in Zusammenarbeit mit dem Stellvertreter des Kommandeurs für politische Arbeit" ${ }^{\text {59 }}$. Dieser hatte die Parteiorganisationen anzuleiten und ihre Arbeit zu organisieren ${ }^{60}$. Die Forderung der Instruktion, dies solle „unter Wahrung der Leninschen Normen des Partei-

52 Ebenda, Bl. 155.

53 Ebenda, Bl. 153.

54 Ebenda.

55 Ebenda, Bl. 159.

56 Innendienstvorschrift DV-10/3, 1963, S. 44.

57 Instruktion für die Parteiorganisationen der SED in der NVA und für die Politorgane der NVA. Protokoll Nr. 39/63 der Sitzung des Politbüros des ZK der SED, 5. November 1963, SAPMO-BArch, DY 30/J IV 2/2/904, Bl. 171.

58 Ebenda, Bl. 157.

59 Ebenda, Bl. 171.

60 Ebenda, Bl. 154, 167. 
lebens" geschehen ${ }^{61}$, stellte letztlich aber einen indirekten Hinweis darauf dar, wie weitreichend der Einfluß der Politstellvertreter auch in den Parteiorganisationen tatsächlich war.

Die Parteiorganisationen, insbesondere die Grundorganisationen der SED in der NVA hatten nun vor allem die Aufgabe, alle Parteimitglieder, aber auch die übrigen Armeeangehörigen ständig zu mobilisieren, etwa „für die weitere Erhöhung der Gefechtsbereitschaft“, die „ununterbrochene Vervollkommnung des eigenen militärischen Könnens" oder die „Festigung der militärischen Disziplin und Ordnung“. Ferner galt es, eine breit angelegte Erziehungsfunktion wahrzunehmen, um beispielweise „hohe politische Wachsamkeit und sozialistische Soldatentugenden, wie Gehorsam, Willensstärke, Mut, Tapferkeit, Entschlossenheit, Kühnheit“ zu vermitteln 62 .

Besonders zeigte sich der Statusverlust der Parteisekretäre im Zusammenhang mit den real verbliebenen Möglichkeiten der Parteiorganisationen, das Verhalten verantwortlicher Offiziere der jeweiligen Einheit zu sanktionieren. Zwar legte die Instruktion erneut fest, daß es die Pflicht der Parteiorganisationen war, „unterschiedslos für alle Mitglieder und Kandidaten, ohne Ansehen der Person, des Dienstgrades und der Dienststellung eine einheitliche Parteidisziplin durchzusetzen“. Auch Offiziere waren dementsprechend zu einer "selbstkritischen Einschätzung ihrer Arbeit“ zu erziehen; denn es galt „Kritik zu entwickeln“ und „Mängel in der Arbeit und im Verhalten jedes Mitgliedes aufzudecken", was die militärische Truppenführung grundsätzlich mit einschloß ${ }^{63}$. Andererseits enthielt die Instruktion jedoch eine entscheidende Einschränkung: Die Durchführung von Parteiverfahren sollte nur dann in der Grundorganisation erfolgen, wenn die Behandlung des Vergehens nicht die "militärische Disziplin oder militärische Geheimhaltung“ gefährden konnte. In einem solchen Fall war das Parteiverfahren durch eine Parteikontrollkommission durchzuführen, wobei diese Entscheidung vom Leiter des zuständigen Politorgans getroffen wurde ${ }^{64}$. So verständlich diese Einschränkung angesichts eines zu erwartenden Autoritätsverlustes der Vorgesetzten bei ihren Untergebenen war, sie stellte letztlich die Ursache dafür dar, daß Parteiverfahren gegen Offiziere außer aus erzieherischen Gründen in Parteiversammlungen in der Regel kaum mehr stattfanden.

In der Truppe führte die gestärkte Stellung der Politstellvertreter und Politorgane offenbar zu gewissen Irritationen. Zumindest sah es der Chef der Politischen Hauptverwaltung Verner im Januar 1964 - bereits zwei Monate nach dem Inkrafttreten der Instruktion - als notwendig an, die Neuerungen zu erläutern, um etwaigen Mißverständnissen vorzubeugen. Er konstatierte, es stehe „keineswegs im Widerspruch“ zu der Forderung nach einer breiten „Entfaltung der innerparteilichen Demokratie“, wenn die Instruktion festlege, daß die Politstellvertreter die Parteiorganisationen „anzuleiten“ hätten ${ }^{65}$. Anscheinend hatten die erweiterten Kompeten-

\author{
61 Ebenda, Bl. 157. \\ 62 Ebenda, Bl. 173. \\ 63 Ebenda, Bl. $174 \mathrm{f}$. \\ 64 Ebenda, Bl. 177. \\ 65 Protokoll Nr. 17/64 der Sitzung der Kreisleitung der SED im Ministerium für Nationale \\ Verteidigung, 3. Januar 1964, BA-MA, VA-P-01/501, Bl. 218.
}


zen der Politstellvertreter jedoch gerade diesen Eindruck erweckt, zumal sie mit einer sichtbaren Entmachtung der Parteisekretäre einhergingen.

Nach Ansicht Verners bestand die Bedeutung der Instruktion jedoch vor allem darin, daß sie die bisher "nicht klar" bestimmten „Pflichten und Rechte“ der Politstellvertreter definierte ${ }^{66}$. Offenbar wollte er zugleich einer möglichen Einschätzung entgegentreten, die Politstellvertreter könnten sich jetzt in einer Art Vorgesetztenverhältnis gegenüber dem Parteikollektiv befinden. Er betonte dagegen, die Instruktion bestimme eindeutig, daß die Anleitung der Parteiorganisationen durch die Politstellvertreter den „Charakter der Tätigkeit eines Instrukteurs des höheren leitenden Politorgans" trage und „unter voller Wahrung der ... Normen des innerparteilichen Lebens" erfolge ${ }^{67}$. Diese zunächst nur intern gemachten Ausführungen wurden anschließend überarbeitet und in einem Grundsatzartikel in der Zeitschrift „Militärwesen“ veröffentlicht ${ }^{68}$, was darauf hindeutet, daß die SED-Führung wegen der gestärkten Stellung der Politoffiziere durchaus negative Folgen für die innere Stabilität des Parteiapparates in der NVA befürchtete.

\section{Der Staatsapparat}

Auch auf der Ebene der staatlichen Institutionen und Instrumente zur Kontrolle des Militärs gab es nach 1961 eine Reihe von Veränderungen. Bevor die SED jedoch 1962 umfassende Neuerungen vor allem auf dem Gebiet des Militärstrafrechts und der Militärjustiz einleitete, kam es nach dem Mauerbau, in dessen Folge unter anderem ein spürbarer Anstieg von Befehlsverweigerungen zu verzeichnen war, zunächst zu einer zeitweiligen Verschärfung der Strafrechtspraxis in der NVA. Diese beendete abrupt die Liberalisierungstendenzen, die sich nach dem Inkrafttreten des Rechtspflegeerlasses vom 30. Januar 1961 in der gesamten DDR-Strafjustiz zunehmend durchgesetzt hatten ${ }^{69}$. Offenbar kam dieser Kurswechsel der SED-Führung sowohl für die Militärstaatsanwaltschaft als auch für die zivilen Strafgerichte, die zu dieser Zeit noch für Militärstraftaten zuständig waren, äußerst unvermittelt. So konstatierte die Hauptabteilung II des Ministeriums der Justiz im September 1961: „Die Urteile aus der Zeit nach dem 13. 8. 1961 zeigen, daß die Gerichte die Gesellschaftsgefährlichkeit der Straftaten gegen die militärische Disziplin noch nicht immer richtig einschätzen und deshalb zum Ausspruch falscher[,] im wesentlichen zu niedriger Strafen kommen. In einer ganzen Reihe von Entscheidungen kommt die in Deutschland gegenwärtig verschärfte Klassenkampfsituation nicht richtig zum Ausdruck." 70

Angesichts der erkennbaren Stabilisierung der DDR nach dem Mauerbau kam es jedoch bereits Anfang 1962 erneut zu einer Entspannung in der Strafpolitik. Diese erfolgte zeitgleich mit der Modifikation der in den Streitkräften gültigen strafrecht-

66 Ebenda.

67 Ebenda.

68 Verner, Für die weitere Stärkung der führenden Rolle der Partei, S. 168.

69 Vgl. Werkentin, Politische Strafjustiz in der Ära Ulbricht, S. $268 \mathrm{ff}$.

70 Vorläufige Einschätzung der Rechtsprechung nach dem 13. August 1961 in Militärsachen, 12. September 1961, SAPMO-BArch, DY 30/IV 2/12/47, Bl. 240. 
lichen Bestimmungen. Es war die Absicht der SED, die gültigen rechtlichen Normen der veränderten militärpolitischen Situation anzupassen und auf diese Weise auch eine Normalisierung der zivil-militärischen Beziehungen zu bewirken. $\mathrm{Zu}$ diesem Zweck wurde als „Zweites Gesetz zur Ergänzung des Strafgesetzbuches“ ein eigenständiges „Militärstrafgesetz“ (MStG) erlassen, das den dritten Teil des Strafrechtsergänzungsgesetzes von 1957 ablöste und am 24. Januar 1962 in Kraft trat $^{71}$.

Durch das neue Militärstrafgesetz sollte eine Erweiterung der im Strafrechtsergänzungsgesetz nur ungenügend spezifizierten Straftatbestände verwirklicht werden. Schon 1959 hatte das Justizministerium auf diesen Mangel hingewiesen und geäußert: „Die Erfahrung in der Arbeit mit den Strafrechtsnormen über Verbrechen und Vergehen gegen die militärische Disziplin hat gezeigt, daß die $\$ \$ 32 \mathrm{ff}$. StEG nicht in jeder Beziehung klar und zweckentsprechend formuliert sind und Lücken enthalten, so daß dadurch in der Praxis zum Teil erhebliche Schwierigkeiten entstanden, die nur mit einer Neufassung beseitigt werden können."72

Neben einer Differenzierung strebte die SED anscheinend auch die Abkehr von der generalklauselartigen Formulierung der Straftatbestände an. Vor dem Hintergrund der Umwandlung der NVA von einer Freiwilligen- in eine Wehrpflichtarmee erwartete man von der weiteren Anwendung der Normen des Strafrechtsergänzungsgesetzes vermutlich eine für die innere Ordnung der Streitkräfte destabilisierende Wirkung. Bis zu diesem Zeitpunkt hatten der SED die Straftatbestände des Strafrechtsergänzungsgesetzes nicht zuletzt als ein Instrumentarium gedient, dem Widerstand des Offizierskorps gegen die politische Durchdringung der NVA wirksam begegnen zu können. Bei der Begründung des Militärstrafgesetzes vor der Volkskammer konstatierte die Justizministerin Hilde Benjamin dementsprechend, das Gesetz resultiere „unmittelbar aus der Einführung der Wehrpflicht“. Im Hinblick auf das Strafrechtsergänzungsgesetz sagte sie: „Diese erste Regelung der Verbrechen gegen die militärische Disziplin im sozialistischen Strafrecht war kurz; sie umfaßte sieben Paragraphen und entsprach den damaligen Bedürfnissen." Dies sei jetzt jedoch nicht mehr genügend ${ }^{73}$.

Das Militärstrafgesetz enthielt insgesamt 30 Bestimmungen: Neben den bereits im Strafrechtsergänzungsgesetz aufgeführten Tatbeständen „Fahnenflucht“ $(\mathbb{S} 4)$ und „Unerlaubte Entfernung“ (\$ 6) war jetzt auch das „Nichtanzeigen der Fahnenflucht“ (\$5) strafbewehrt. Als neue Delikte wurden „Dienstentziehung und Dienstverweigerung“ (\$7) sowie „Feigheit vor dem Feind“ $(\$ 8)$ in das Militärstrafgesetz aufgenommen. Die Straftatbestände „Befehlsverweigerung“ (\$9) und „Nichtdurchführung eines Befehls" $(\mathbb{S 1 0})$, die im Strafrechtsergänzungsgesetz noch in einem Paragraphen zusammengefaßt waren, zerfielen in zwei gesonderte Ungehorsamstatbestände. Das Delikt „Angriff auf Vorgesetzte, Wachen oder Streifen“ (\$11) stellte eine Erweiterung der bisherigen Regelung um die letzten beiden Personenkreise dar. Straftaten gegen die militärische Disziplin und Ordnung waren zudem

71 Vgl. Buth, Die Entwicklung des militärischen Befehlsrechts, S. 151.

72 Begründung der vorgeschlagenen Strafrechtsnormen über Verbrechen und Vergehen gegen die Kampfkraft der Organe der Landesverteidigung der DDR, 25. April 1959, SAPMOBArch, DY 30/IV 2/12/47, Bl. 21.

73 Benjamin, Neue Bestimmungen zum Schutz der Kampfkraft, S. 109. 
„Beleidigung Vorgesetzter oder Unterstellter“ $(\$ 12)$ sowie „Mißbrauch der Dienstbefugnisse und Verletzung der Dienstpflichten“ (\$13), wobei Paragraph 13 gegenüber dem Strafrechtsergänzungsgesetz inhaltlich um den zweiten Tatbestand ergänzt worden war. Darüber hinaus enthielt das Militärstrafgesetz als Straftatbestände „Verletzung des Beschwerderechts“ ( $(14)$, „Verletzung militärischer Geheimnisse“ $(\$ 15)$, „Verletzung der Vorschriften über den Wachdienst“ $(\mathbb{1} 16)$, „Verletzung der Vorschriften des funktechnischen und Bereitschaftsdienstes“ (\$17), „Verletzung der Vorschriften über den Grenzdienst“ (\$18), „Verletzung der Vorschriften über den Flugbetrieb“ ( $(19)$, „Beeinträchtigung der Einsatzbereitschaft der Kampftechnik und militärischen Ausrüstung“ $(\mathbb{\$} 20)$ sowie „Verletzung der Meldepflicht" $(\$ 21)^{74}$.

Nach Aussage des Militärjuristen und späteren Präsidenten des Obersten Gerichtes der DDR, Günter Sarge, hatte das Militärstrafgesetzbuch zwei Hauptfunktionen: zum einen die „Abwehr und rücksichtslose Bekämpfung der sich in schwerwiegenden Militärstraftaten widerspiegelnden Angriffe des Gegners auf die Kampfkraft der Armee und Bekämpfung schwerer Verstöße einzelner rückständiger Armeeangehöriger auf die militärische Disziplin und Ordnung" und zum anderen die „Beseitigung bürgerlicher Denk- und Lebensgewohnheiten bei zurückgebliebenen Armeeangehörigen, wie Disziplinlosigkeit, Ungehorsam und andere negative Verhaltensweisen" sowie „die Erziehung zum sozialistischen Bewußtsein"75.

Die stärkere Betonung des Erziehungsgedankens und die Differenzierung der Straftatbestände im Militärstrafgesetz fügten sich nahtlos in die von der SED 1962/1963 postulierte rechtspolitische Linie ein. Im Zuge der Konsolidierung des Regimes sollte jetzt anstelle der Repressiv- die Erziehungsfunktion der Rechtsprechung in den Vordergrund treten ${ }^{76}$. In der Phase des "umfassenden Aufbaus des Sozialismus" nach „Überwindung des Klassenantagonismus" in der DDR war es nach Auffassung der Partei aufgrund der „zunehmenden bewußten Einhaltung des sozialistischen Rechts“ durch die Bevölkerung nunmehr möglich, die „Einheit von Recht und Erziehung" zu vertiefen. Die Funktion des Rechts sollte jetzt hauptsächlich auf die Veränderung der gesellschaftlichen Beziehungen und der Menschen selbst zielen. Juristischer Zwang wurde daher lediglich als Hilfsmittel und als Hebel zur Herbeiführung des bewußten Handelns begriffen ${ }^{77}$. Trotz dieser Linie lag die Strafandrohung des Militärstrafgesetzes zumeist jedoch über der des Strafrechtsergänzungsgesetzes $^{78}$. Da nur dessen drittes Kapitel aufgehoben wurde, galt zudem auch weiterhin der materielle Verbrechensbegriff 79 .

74 Weiterhin wurden vier Delikte in das Gesetz aufgenommen, die ausschließlich im Fall eines Kampfeinsatzes der NVA Bedeutung hatten. Diese waren die „Schändung Gefallener und Mißbrauch der Lage Verwundeter" $(\$ 22)$, die "Gewaltanwendung und Plünderung im Kampfgebiet“ (\$23) sowie die „Verletzung der Rechte der Kriegsgefangenen“ (\$24) und die "Verletzung der Zeichen des Roten Kreuzes“ (\$25). Zweites Gesetz zur Ergänzung des Strafgesetzbuches. - Militärstrafgesetz -. Vom 24. Januar 1962, GBl. I 1962, S. $25 \mathrm{ff}$.

75 Sarge, Das Militärstrafgesetz vom Januar 1962, S. 526.

76 Vgl. Böckenförde, Die Rechtsauffassung im kommunistischen Staat, S. $78 \mathrm{ff}$.; Schuller, Geschichte und Struktur des politischen Strafrechts der DDR, S. 400 ff.; Werkentin, Politische Strafjustiz in der Ära Ulbricht, S. $268 \mathrm{ff}$.

77 Thesen zum Wesen und zur Entwicklung des sozialistischen Rechts, S. $1841 \mathrm{ff}$.

78 So konnten gemäß $₫ 26$ Militärstrafgesetz Militärstraftaten der Paragraphen 4 Absatz 3, 7 Absatz 3, 8 Absatz 1 und 2, 9 Absatz 3, 11 Absatz 2 und 4, 16 Absatz 2 b, 17 Absatz 2 b, 22 
Die Hervorhebung der Erziehungsfunktion des Rechts im Anschluß an den Mauerbau fand ihren sichtbaren Ausdruck im Erlaß des Staatsrates über die grundsätzlichen Aufgaben und die Arbeitsweise der Organe der Rechtspflege vom 4. April 196380, auf dessen Grundlage die SED die weitere Umorientierung des Strafensystems betrieb. Mit Ausnahme von Staatsverbrechen sollten Freiheitsstrafen an Bedeutung verlieren und statt dessen Erziehung und Wiedereingliederung der Täter im Vordergrund stehen. Nicht die Bekämpfung, sondern die Aufdeckung und Untersuchung der gesellschaftlichen Ursachen von Kriminalität hatte jetzt im Mittelpunkt der Gerichtsverfahren zu stehen, weshalb die Mitwirkung gesellschaftlicher Organe und Kräfte an diesen verstärkt werden sollte ${ }^{81}$. Der Rechtspflegeerlaß forderte dementsprechend die „Erweiterung der unmittelbaren Teilnahme der Werktätigen an der Rechtsprechung und der Erziehung Gestrauchelter sowie die verstärkte kollektive Selbsterziehung der Bürger durch gesellschaftliche Organe der Rechtspflege" 82.

Dieser neue Ansatz wurde auch auf dem Gebiet der Militärjustiz verwirklicht. Dabei übernahmen in der NVA die jeweiligen militärischen Kollektive die Funktion der Schieds- bzw. Konfliktkommissionen im zivilen Bereich ${ }^{83}$. Durch die „Militärgerichtsordnung" vom 4. April $1963^{84}$ wurde zudem die Tätigkeit von Militärschöffen eingeführt. Diese wirkten zum einen als Richter an den ebenfalls neugeschaffenen Militärgerichten. Zum anderen oblagen ihnen in der NVA Erziehungsaufgaben in Rechtsfragen ${ }^{85}$. Die Militärschöffen waren Angehörige der NVA oder der Organe des Wehrersatzdienstes, die in den Einheiten und Dienststellen für zwei Jahre in ihre Funktion gewählt wurden ${ }^{86}$. In den Verhandlungen vor den Militärgerichten und den Verhandlungen erster Instanz vor den Militärobergerichten nahmen neben dem jeweiligen Militärrichter jeweils zwei Militärschöffen teil ${ }^{87}$, die das „Richteramt in vollem Umfange und mit gleichem Stimmrecht wie die Militärrichter" ausübten ${ }^{88}$. In der Truppe selbst waren die Militärschöffen im Rahmen ihres Erziehungsauftrages „an der vorbeugenden Tätigkeit“ der Militärgerichte sowie „an der Propagierung und Durchsetzung des sozialistischen Rechts" beteiligt. Sie hatten die Armeeangehörigen zur Wahrung der sozialistischen Gesetzlichkeit zu „erziehen" ${ }^{\text {"9 }}$. Dies geschah etwa "durch die Erläuterung der Gesetze und durch die Auswertung geeigneter Verfahren“, die an den Militärgerichten verhandelt wurden ${ }^{0}$.

und 23 ,in besonders schweren Fällen mit lebenslangem Zuchthaus oder mit dem Tode bestraft werden“. Militärstrafgesetz, GBl. I 1962, S. 27.

79 Buth, Die Entwicklung des militärischen Befehlsrechts, S. 153.

80 Erlaß des Staatsrates der Deutschen Demokratischen Republik über die grundsätzlichen Aufgaben und die Arbeitsweise der Organe der Rechtpflege. Vom 4. April 1963, GBl. I 1963, S. $21 \mathrm{ff}$.

81 Vgl. Böckenförde, Die Rechtsauffassung im kommunistischen Staat, S. $79 \mathrm{ff}$.

82 Erlaß des Staatsrates, GBl. I 1963, S. 24.

83 Vgl. Kap. VIII.4. dieser Studie.

84 Erlaß des Staatsrates der Deutschen Demokratischen Republik über die Stellung und die Aufgaben der Gerichte für Militärstrafsachen. (Militärgerichtsordnung).Vom 4. April 1963, GBl. I 1963, S. $71 \mathrm{ff}$.

85 Hatzius, Grundlagen und Entwicklung der Militärstrafgerichtsbarkeit, S. $88 \mathrm{ff}$.

86 Militärgerichtsordnung, GBl. I 1963, S. 73.

87 Ebenda, S. $74 \mathrm{f}$.

88 Ebenda, S. 72.

89 Stellung, Aufgaben und Wahl der Militärschöffen (Militärschöffenordnung). Anordnung 
Die Bedeutung, die diese Institution in kurzer Zeit erlangte, läßt sich daran ablesen, daß die 1304 Militärschöffen, die in der NVA 1963 in 143 Militärschöffenkollektiven tätig wurden, im Rahmen ihrer Erziehungsarbeit in den Einheiten unter anderem 1300 „vorbeugende Maßnahmen“ durchführten. Dies waren Auswertungen, Vorträge und Aussprachen, an denen insgesamt 49572 Armeeangehörige teilnahmen. Die SED stellte Anfang 1965 fest, die Militärschöffen hätten „die in sie gesetzte Erwartung voll erfüllt“. Vor allem habe „ihre unmittelbare Kenntnis der militärischen Belange" in den Verhandlungen an den Militärgerichten eine "gründliche und sachbezogene Aufklärung des Sachverhaltes und Einschätzung der Person" gewährleistet. In Zukunft, so forderte man, sollten die Militärschöffen „als Bindeglied“ zwischen den Militärgerichten und Kommandeuren „die Unterstützung der militärischen Kollektive bei der kollektiven Selbsterziehung verstärken"91.

Die Schaffung der Militärgerichte ${ }^{92}$ auf der Grundlage des Gesetzes zur Ergänzung des Gerichtsverfassungsgesetzes der DDR vom 24. Januar $1962^{93}$ erfolgte

Nr. 7/63 des Ministers für Nationale Verteidigung, 4. April 1963, BA-MA, VA-01/13993, Bl. 27.

90 Militärgerichtsordnung, GBl. I 1963, S. 72.

91 Bericht über die Militärschöffentätigkeit in der ersten Wahlperiode, 20. März 1965, SAPMO-BArch, DY 30/IV A2/12/51, o. Pag.

92 Umstritten ist in der Literatur die Frage, ob die Militärgerichte als „Sondergerichte " zu qualifizieren seien. Schon 1963 ist einer derartigen Sicht von Seiten dér SED offiziell widersprochen worden. Es hieß, die Militärgerichte seien „keine Sondergerichte, sondern ,Gerichte für besondere Sachgebiete“ im Sinne des Artikels 134 der Verfassung“ der DDR, die „auf der Grundlage des Gerichtsverfassungsgesetzes, der Militärgerichtsordnung sowie der Strafprozeßordnung vom 2. 8. 1958“ arbeiteten. Zudem sei kein „selbständiges zentrales Organ“, wie dies beim sogenannten Reichskriegsgericht der Fall gewesen sei, „für die höchstrichterliche Rechtsprechung in Militärstrafsachen" zuständig. Vielmehr obliege auch diese dem Obersten Gericht der DDR. Ruf/Hartmann, Militärgerichte - neue sozialistische Rechtspflegeorgane, S. 831, 833. Nach Ansicht von Berthold Hatzius waren die Militärgerichte dagegen „selbständige Gerichte“, die „in jeder Instanz von den allgemeinen Strafgerichten völlig getrennt" waren und „nur der allen Gerichten der DDR gemeinsamen höchsten gerichtlichen Instanz, dem Plenum des Obersten Gerichts" unterstanden. Hatzius, Grundlagen und Entwicklung der Militärstrafgerichtsbarkeit, S. 80. Erik Sündram wiederum hat kurz vor der Auflösung der DDR die Auffassung vertreten, es habe sich bei den Militärgerichten zwar um keine "Sondergerichte" gehandelt. Andererseits hätten sie sich aber offensichtlich weder in der NVA noch in der Bevölkerung als „anerkannt-öffentliche Spruchkörper etablieren“ können. Letztlich hätten sie als "Strafgerichte“ gegolten und seien als „repressiv verfemt" gewesen. Sündram, Die Sondergerichte, S. 5. Zuletzt hat unter anderem Peter Joachim Lapp geäußert, die Militärgerichte hätten zwar im Gerichtssystem der DDR eine „Sonderstellung" eingenommen. Sie könnten aber nicht als Sondergerichte bezeichnet werden, da es „keine besonderen Verfahrensprinzipien“ gegeben habe und die „maßgeblichen Rechtsvorschriften wie Strafgesetzbuch und Strafprozeßordnung" auch für sie verbindlich gewesen seien. Lapp, Die Nationale Volksarmee 1956-1990, S. 1942. Diese Tatsachen dürften für die Beurteilung der Militärgerichte jedoch nicht allein ausschlaggebend sein. Wichtig ist darüber hinaus, daß wegen der fehlenden Gewaltenteilung in der DDR auch an den Militärgerichten keine richterliche Unabhängigkeit existierte und die Rechtsprechung letztlich nach Maßgabe der SED erfolgte. Letzteres dürfte durch die weitgehende institutionelle Trennung der Militärgerichtsbarkeit vom übrigen Gerichtssystem der DDR noch erleichtert worden sein. Aus diesen Gründen verfügten die Militärgerichte, wenn auch nicht formal, so jedoch faktisch über einen signifikanten Sonderstatus.

93 Gesetz zur Ergänzung des Gerichtsverfassungsgesetzes der Deutschen Demokratischen Republik. Vom 24. Januar 1962, GBl. I 1962, S. 28. 
nach Angaben des Nationalen Verteidigungsrates „in Anlehnung an die gesetzlichen und praktischen Erfahrungen der Militärgerichtsbarkeit der UdSSR und der Volksrepublik Polen“94. Die Entscheidung der SED, spezielle Gerichte für Militärstrafsachen zu bilden, dürfte aber auch darauf zurückzuführen sein, daß sich die bis dahin geübte Praxis als ineffizient erwiesen hatte. Die ZK-Abteilung für Staats- und Rechtsfragen stellte diesbezüglich im September 1961 fest, die Strafkammern der Kreisgerichte und die Strafsenate der Bezirksgerichte, die die Militärstrafsachen bearbeiteten, hätten zu diesen „keine richtigen Beziehungen“. Zum Teil bestehe offensichtlich die Vorstellung, „Militärstrafsachen seien besondere Sachen und deshalb besonders zu behandeln" "95. Zusätzlich betrachtete anscheinend die Oberste Staatsanwaltschaft die Militärstaatsanwaltschaft „nicht als Bestandteil der einheitlichen Staatsanwaltschaft der DDR“, so daß in der Militärstaatsanwaltschaft wegen der ungenügenden Aufsicht ebenfalls die Auffassung vorherrschte, es würden „für den Bereich der Armee Besonderheiten bei der Strafverfolgung gelten“. Im Ergebnis, so konstatierte man, verfüge der Leiter der Militärstaatsanwaltschaft gegenüber den anderen Zweigen der Staatsanwaltschaft über „eine Sonderstellung“96. Eine der prinzipiellen Schlußfolgerungen lautete daher, es solle „geprüft werden, ob jetzt nicht der Zeitpunkt herangereift" sei, „wo an die Vorbereitung der Bildung von Militärgerichten geschritten werden " könne"

Durch die Militärgerichtsordnung von 1963 wurde festgelegt, daß die „Rechtsprechung in Strafsachen gegen Militärpersonen und gegen Teilnehmer an Straftaten, die gegen die militärische Sicherheit gerichtet" waren, von den neugeschaffenen Gerichten für Militärstrafsachen ausgeübt werden sollte, die „als Teile des Gerichtssystems" der DDR arbeiteten ${ }^{98}$. Als oberstes Militärgericht fungierte ein beim Obersten Gericht der DDR eingerichtetes „Kollegium für Militärstrafsachen“. Vergleichbar mit den Bezirksgerichten waren die auf der mittleren Ebene gebildeten Militärobergerichte. Die Militärgerichte der unteren Ebene entsprachen den Kreisgerichten ${ }^{99}$.

Auch in bezug auf die Tätigkeit der Hauptabteilung I des MfS in den Streitkräften gab es nach dem Mauerbau 1961 einige organisatorische Neuerungen. Die ZKAbteilung für Sicherheitsfragen hatte schon Anfang des Jahres gefordert, zwischen der Politischen Verwaltung des Ministeriums für Nationale Verteidigung und der Hauptabteilung I des MfS müsse eine „Aussprache“ über die „Prinzipien und Methoden der Zusammenarbeit" stattfinden ${ }^{100}$. Im Zuge der Konsolidierung der NVA kam es zwar offenbar zu keiner Verminderung der Kompetenzen der im Ministe-

94 Bericht über den Stand des Aufbaues der Militärgerichte der DDR. Protokoll der 12. Sitzung des Nationalen Verteidigungsrates der DDR, 14. September 1962, BA-MA, DVW $1 / 39469, \mathrm{Bl} .38$. Eckart Busch hat in diesem Zusammenhang darauf hingewiesen, daß auch ein „Rückgriff auf Vorschriften des ,Militärstrafgesetzbuches für das Deutsche Reich' vom 20. 6. 1872 ... unverkennbar" sei. Busch, Das Militärstrafrecht der SBZ, S. 156.

95 Schlußfolgerungen zur Verbesserung der Arbeit der Militäroberstaatsanwaltschaft, 28. Oktober 1961, SAPMO-BArch, DY 30/IV 2/12/47, Bl. 275.

96 Ebenda.

97 Ebenda., Bl. 277.

98 Militärgerichtsordnung, GBl. I 1963, S. 71.

99 Ebenda, S. $73 \mathrm{ff}$.

100 Analyse der Feindarbeit im Jahre 1960 unter besonderer Berücksichtigung des IV. Quartales, 1961, SAPMO-BArch, DY 30/IV 2/12/47, Bl. 170. 
rium als Verwaltung 2000 firmierenden Hauptabteilung I in der militärischen Aufklärungs- und Abwehrarbeit. Insofern unterlag die militärische Truppenführung wie in den fünfziger Jahren der intensiven Überwachung durch das MfS, deren Effizienz nicht zuletzt auf dem verzweigten IM-Netz basierte. Veränderungen ergaben sich aber infolge der erkennbaren Absicht der SED, die Stellung der Verwaltung 2000 in der NVA auf formaler Grundlage umfassend zu regeln. Offenbar zielte die Parteiführung im Zuge des 1962 vollzogenen Kurswechsels in der Strafrechtspflege darauf ab, die Tätigkeit der MfS-Mitarbeiter auch in den Streitkräften einer allgemeinen Normierung zu unterziehen.

Denn wie aus einem an Ulbricht gerichteten „Bericht über die Arbeit des MfS“ aus demselben Jahr hervorgeht, scheint die Untersuchungspraxis des Staatssicherheitsdienstes in der SED-Führung zunehmend auf Kritik gestoßen zu sein. In dem Bericht hieß es, daß „Gesetzverletzungen bei der Durchführung der Ermittlungsund Untersuchungsarbeit" und aufgrund der "gesellschaftlichen Entwicklung heute nicht mehr notwendig“ seien und „zu Störungen der Beziehungen mancher Bürger zu unserem Staat führen" könnten. Daher solle die Verhaftung von Beschuldigten in Zukunft „in der Regel auf Grundlage eines richterlichen Haftbefehls erfolgen“. Laut dem Bericht war es aber nach wie vor üblich, daß „die beschuldigten Personen größtenteils ohne richterlichen Haftbefehl festgenommen " wurden, und dieser „erst nach der Festnahme“ erwirkt wurde. Kritisiert wurde auch die „Beeinflussung der Inhaftierten durch Mitarbeiter der Untersuchungsorgane auf den Verzicht der Anwendung von Rechtsmitteln"101.

Im übrigen moniert man, daß die Staatsanwälte zu wenig die „Aufsicht über laufende Vorgänge“ führten. Zumeist beginne ihre Arbeit erst, „wenn das Verfahren seitens des Untersuchungsorgans abgeschlossen“ sei und der Schlußbericht vorliege ${ }^{102}$. Dies widersprach der Pflicht der Staatsanwälte, über alle Untersuchungshandlungen - so auch über die des MfS - die Aufsicht auszuüben. Ferner heißt es im Bericht, es gebe in der Praxis „keine klare Abgrenzung“ zwischen den Untersuchungsorganen des Ministeriums des Innern und des MfS bei der Durchführung von Ermittlungs- und Untersuchungsverfahren. In diesem Zusammenhang wurde darauf hingewiesen, daß in den Verantwortungsbereich des MfS allein die Staatsverbrechen gemäß der Paragraphen 13-26 des Strafrechtsergänzungsgesetzes von 1957 und die Militärstraftaten laut Militärstrafgesetz von 1962 fielen ${ }^{103}$.

Obwohl sich der Bericht auf das MfS im ganzen bezog, ist anzunehmen, daß die aufgeführten Kritikpunkte im einzelnen genauso auf die Hauptabteilung I zutrafen. In Anbetracht der erreichten Stabilisierung des SED-Regimes sah die Parteiführung die bewußte Mißachtung rechtlicher Normen und Gesetze durch das MfS, die vor dem Mauerbau offenbar üblich gewesen und akzeptiert worden war, jetzt nicht mehr als opportun, innenpolitisch sogar als kontraproduktiv an.

101 Bericht über die Arbeit des MfS, 1962, SAPMO-BArch, DY 30/J IV 2/202/62, o. Pag.

102 Allein in den Jahren 1961 und 1962 wurden von Seiten des MfS gegen 5593 Militärpersonen Ermittlungsverfahren durchgeführt. Im Zeitraum von Januar 1961 bis November 1965 waren es insgesamt 13773. Darunter befanden sich als Delikte unter anderem 196 Fälle von Staatsgefährdender Hetze und Propaganda, 2339 Fälle von Fahnenflucht und 209 Fälle von Staatsverleumdung. Statistische Übersicht über Straftaten von Militärpersonen in den Jahren 1961-1965, 24. Dezember 1965, BStU, ZA, MfS HA IX 11964/MF, BI. 1 f.

103 Bericht über die Arbeit des MfS, 1962, SAPMO-BArch, DY 30/J IV 2/202/62, o. Pag. 
Es dauerte jedoch bis zum 15. November 1963, bis eine formale „Vereinbarung über die Zusammenarbeit und das Zusammenwirken der Organe des Ministeriums für Staatssicherheit und des Ministeriums für Nationale Verteidigung " getroffen wurde ${ }^{104}$. Mit dem Ziel einer "Zusammenfassung bestehender Einzelregelungen“" und einer klaren „Abgrenzung der Aufgaben und der gegenseitigen Befugnisse“ sollte die Zusammenarbeit der beiden Ministerien verbessert werden. Gemäß der Vereinbarung bestand die Aufgabe der Verwaltung 2000 in der „Gewährleistung der staatlichen Sicherheit in der Nationalen Volksarmee“. Im Rahmen der Abwehr kam ihr vor allem die Aufgabe zu, „Pläne und Absichten des Gegners, die gegen die Nationale Volksarmee gerichtet“ waren, „aufzuklären und Maßnahmen zu ihrer Verhinderung zu ergreifen“. Sie hatte „Spione, Agenten und andere staatsfeindliche Elemente in der Nationalen Volksarmee zu entlarven und unschädlich zu machen". Auf dem Gebiet der Aufklärung bestimmte die Vereinbarung als Aufgabe, „das Vorfeld und die Tiefe des gegnerischen Territoriums aufzuklären“ und hierbei „die Tätigkeit des Gegners in der Grenzzone der DDR, insbesondere im 500- bzw. 100-m-Schutzstreifen, aufzuklären, zu bekämpfen und zu liquidieren"105.

Die Verwaltung 2000 gliederte sich entsprechend den militärischen Ebenen in Abteilungen, Unterabteilungen, Operativgruppen und Offiziere für "Abwehr“ oder „Aufklärung“ bei den Kommandos der Teilstreitkräfte und Militärbezirke sowie bei der Stadtkommandantur Berlin und bei den Stäben der Verbände, Truppenteile, Grenzbataillone ${ }^{106}$ sowie den Dienststellen der NVA ${ }^{107}$. Die Mitarbeiter der

104 Frank Petzold hat angenommen, daß infolge dieser Vereinbarung das Unterstellungsverhältnis der Mitarbeiter der Verwaltung 2000 sowohl in den Grenztruppen als auch in der gesamten NVA geändert worden sei. Nunmehr hätten die MfS-Offiziere „bei ihrer Tätigkeit das militärische Vorgesetztenverhältnis und die Verantwortung der NVA-Kommandeure zwar noch zu ,beachten" gehabt, „sich diesen aber keinesfalls verbindlich fügen" müssen. Petzold, Der Einfluß des MfS auf das DDR-Grenzregime, S. $145 \mathrm{f}$. Tatsächlich handelte es sich bei der Vereinbarung zwischen den Ministerien für Nationale Verteidigung und Staatssicherheit aber wohl in erster Linie um die formale Zusammenfassung von Einzellregelungen, die zumeist schon seit den fünfziger Jahren existierten. Vgl. S. $122 \mathrm{ff}$.

105 Vereinbarung über die Zusammenarbeit und das Zusammenwirken der Organe des Ministeriums für Staatssicherheit und des Ministeriums für Nationale Verteidigung, 15. November 1963, BA-MA, AZN/32572, Bl. $5 \mathrm{ff}$.

106 Als Folge der Eingliederung der Grenztruppen in die NVA im September 1961 existierten zunächst zwei Abschirmdienste in den Streitkräften: neben der Verwaltung 2000 war dies der ursprünglich eigenständige Aufklärungsdienst der ehemaligen Grenzpolizei. Obwohl er zu diesem Zeitpunkt offenbar bereits umfassend vom MfS unterwandert worden war, war er diesem jedoch formal nicht unterstellt. Vgl. Petzold, Der Einfluß des MfS auf das DDR-Grenzregime, S. 140ff. Mielke gelang es im Anschluß innerhalb eines Vierteljahres, dieses Unterstellungsverhältnis zugunsten des MfS zu verändern. Gemäß seinem Befehl vom 10. Dezember 1961 wurden die „Aufklärungsorgane der Grenztruppen, ... aus der Unterstellung, [dem] Struktur- und Stellenplan der Grenztruppen der Nationalen Volksarmee herausgelöst" und dem Ministerium für Staatssicherheit unterstellt. Befehl Nr. 598/61 des Ministers für Staatssicherheit, 10. Dezember 1961, BStU, ZA, MfS BdL/Dok. Nr. 000592, Bl. 1. Unklar bleibt aber, ob er dabei auf Widerstand des Ministeriums für Nationale Verteidigung stieß. Zumindest sah es Mielke als geboten an, in einem weiteren Befehl vom 19. Januar 1962 die Gültigkeit des neuen Unterstellungsverhältnisses nochmals zu bekräftigen. Dort heißt es: „Die Abteilung Aufklärung beim Kommando Grenze der NVA befindet sich mit Wirkung vom 10.12. 1961 im Befehls- und Unterstellungsverhältnis der Hauptabteilung -I- des Ministeriums für Staatssicherheit.... Die Mitarbeiter der Diensteinheiten der Abteilung Aufklärung beim Kommando Grenze der NVA ... sind Angehörige 
Verwaltung 2000 trugen im Dienst die Uniform ihrer jeweiligen Einheit ${ }^{108}$, waren jedoch Angehörige des Ministeriums für Staatssicherheit und den NVA-Kommandeuren ihrer jeweiligen Einheit „weder unterstellt noch rechenschaftspflichtig“109. Der Minister für Nationale Verteidigung selbst hatte keinerlei Einfluß auf die Stellenpläne der Verwaltung 2000. Diese wurden ihm nur „zur Kenntnisnahme übersandt" ${ }^{\prime 10}$. Trotz ihrer Unterstellung unter das MfS hatten die Offiziere der Verwaltung 2000 entsprechend der Vereinbarung bei ihrer Tätigkeit die „Verantwortung der Kommandeure ... als militärische Vorgesetzte und Einzelleiter" zu achten. Auch waren sie dazu verpflichtet, die militärische Disziplin und Ordnung zu wahren und sich streng an die in ihrem Verantwortungsbereich geltenden militärischen Bestimmungen zu halten ${ }^{111}$.

Der Chef der Verwaltung 2000 sollte eine „enge dienstliche Verbindung" sowohl zum Minister für Nationale Verteidigung als auch zum Chef der Politischen Hauptverwaltung und dem Chef des Hauptstabes unterhalten und diese informieren. Er hatte das Recht, „an allen Sitzungen des Kollegiums des Ministeriums für Nationale Verteidigung mit beratender Stimme teilzunehmen"112. Die Abwehr- und Aufklärungsoffiziere in der Truppe waren ihrerseits dazu verpflichtet, die Kommandeure, Chefs und Leiter in der NVA über die Erkenntnisse und Ergebnisse zu informieren, die sie bei ihrer Arbeit gewonnen hatten. Die „Regeln der Konspiration“ waren jedoch „streng zu beachten und einzuhalten "113. Im Gegenzug sollten die Mitarbeiter der Verwaltung 2000 bei ihrer Arbeit von den Kommandeuren und auch den übrigen Angehörigen der NVA unterstützt werden ${ }^{114}$.

Die Vereinbarung zwischen dem MfS und dem Ministerium für Nationale Verteidigung gestattete den Mitarbeitern der Verwaltung 2000 den „Zugang zu allen Stellen in ihrem Verantwortungsbereich". Sie hatten das Recht, Armeeangehörige „ohne vorheriges Einverständnis des jeweiligen Kommandeurs ... zu Aussprachen und Vernehmungen zu bestellen“, wobei aber die militärischen Bestimmungen einzuhalten waren. Für die Vernehmung von Kommandeuren vom Bataillonskommandeur an aufwärts und von Stabsoffizieren bedurfte es jedoch einer „vorherigen Bestätigung" durch den Chef der Verwaltung 2000. Ausdrücklich wurde jetzt festgelegt, daß die Festnahme von NVA-Angehörigen den Kommandeuren ab Divisionskommandeur "unmittelbar nach erfolgter Durchführung bekanntzugeben“ war. Zudem sollte der Minister für Nationale Verteidigung vom Chef der Verwaltung 2000 ,in der Regel vor der Festnahme“ in Kenntnis gesetzt werden ${ }^{115}$.

des Ministeriums für Staatssicherheit." Befehl Nr. 56/62 des Ministers für Staatssicherheit, 19. Januar 1962, BStU, ZA, MfS BdL/Dok. Nr. 000813, Bl. 1.

107 Vereinbarung über die Zusammenarbeit und das Zusammenwirken der Organe des Ministeriums für Staatssicherheit und des Ministeriums für Nationale Verteidigung, 15. November 1963, BA-MA, AZN/32572, Bl. 6.

108 Ebenda, Bl. 10.

109 Ebenda, Bl. 6.

110 Ebenda.

111 Ebenda, Bl. 9 f.

112 Ebenda, Bl. 8.

113 Ebenda, Bl. 8 f.

114 Ebenda, Bl. 8.

115 Ebenda, Bl. 9. 
Zusätzlich zu dieser Vereinbarung legte das Politbüro im Rahmen der am 25. Juni 1965 beschlossenen „Grundsätze des Führungssystems im Verteidigungszustand“ auch die Aufgaben des MfS für diesen Fall fest und grenzte sie von denen der NVA ab. In den Zuständigkeitsbereich des MfS im Kriegsfall fiel die Aufrechterhaltung der inneren Sicherheit und Ordnung. Im Hinblick auf die Ministerien des Innern und für Nationale Verteidigung betraf dies ,alle Fragen der Bekämpfung der subversiven Handlungen des Gegners mit spezifischen Mitteln, der Bekämpfung von Staatsverbrechen und anderer Verbrechen“, die auf die „Lähmung der Verteidigungsfähigkeit“ der DDR gerichtet waren. Zudem oblag dem MfS die „Koordinierung der Aufgaben der Rechtspflegeorgane des Ministeriums für Staatssicherheit und des Ministeriums des Innern, insbesondere aller strafrechtlichen Untersuchungshandlungen“. Darüber hinaus war das MfS einerseits zuständig für „alle Fragen der Außenaufklärung und andere einschlägige aktive Maßnahmen“, die mit der militärstrategischen Aufklärung des Ministeriums für Nationale Verteidigung zu koordinieren waren; andererseits fielen auch „alle Fragen der inneren Sicherheit“ der im Bereich des Oberkommandos der Vereinten Streitkräfte eingesetzten Verbände und Truppenteile der NVA und ihrer „territorialen Einheiten“" in das Aufgabengebiet des MfS sowie „alle Fragen der Sicherheit und Ordnung im Operationsgebiet der Verbände und Truppenteile der Nationalen Volksarmee" entsprechend der gemeinsamen Planung des Oberkommandos der Vereinten Streitkräfte. Durch die ergänzende Bestimmung, die Kräfte des MfS seien den zuständigen NVA-Kommandeuren im Operationsgebiet "direkt unterstellt“, wird erkennbar, daß dies außerhalb dieses Bereiches offensichtlich nicht der Fall war ${ }^{116}$.

Das ist ein deutlicher Hinweis auf den weitreichenden Handlungsspielraum des MfS im Falle eines von der DDR geführten bewaffneten Konfliktes. Für diese Annahme spricht zudem, daß das MfS laut Politbürobeschluß für „alle Fragen der inneren Sicherheit in Haft-, Kriegsgefangenen- und Internierungslagern “ zuständig war ${ }^{117}$, ebenso wie für deren „Einrichtung, Verwaltung und Betreuung "118. Insbesondere die Tatsache, daß die Aufsicht über die Kriegsgefangenenlager nicht in den Kompetenzbereich der NVA fiel, zeigt, daß die SED trotz der Konsolidierung und Professionalisierung des Militärs auch 1965 nicht dazu bereit war, der militärischen Truppenführung in Kernbereichen, die sie als besonders sicherheitsrelevant betrachtete, mehr Verantwortung und Autonomie einzuräumen.

\section{Das Militär}

Ab 1961 bestand in der NVA-Spitze und auch von Seiten der SED das erkennbare Bemühen, die Stellung der militärischen Leitungen und insbesondere der Kommandeure zu stärken. Dies geschah vor allem in der Absicht, die Effizienz der militärischen Führungstätigkeit zu verbessern. Aus diesem Grund wurde die Einzelleitung

116 Grundsätze des Führungssystems im Verteidigungszustand. Protokoll Nr. 25/65 der außerordentlichen Sitzung des Politbüros des ZK der SED, 25. Juni 1965, SAPMO-BArch, DY 30/J IV 2/2/993, Bl. $11 \mathrm{f}$.

117 Ebenda, Bl. 13.

118 Ebenda, Bl. 15. 
als gültiges Führungsprinzip in den Streitkräften wieder in erster Linie nach militärischen Gesichtspunkten definiert.

So war etwa ein Regimentskommandeur gemäß der Innendienstvorschrift DV-10/3 von 1959 dazu verpflichtet gewesen, die „Einheit der politischen und militärischen Führung in Zusammenarbeit mit der Parteileitung zu verwirklichen"119. Die neue Innendienstvorschrift, die am 1. Januar 1963 in Kraft trat, wich jedoch entscheidend von diesem Schema ab. Sie betonte vielmehr die unabhängige Entscheidungskompetenz des Kommandeurs als „Einzelleiter“, der jetzt über die „ungeteilte Kommando- und Befehlsgewalt über die ihm Unterstellten“ verfügen sollte. Eine direkte Beteiligung der Parteiorganisationen an der militärischen Entschlußfassung sah die Vorschrift nicht mehr vor. Gefordert wurde allein, daß sich der Kommandeur auf die Parteiorganisationen „stützen“ und „ihren Einfluß“ auf die erfolgreiche Erfüllung der Aufgaben der Einheit voll ausnutzen sollte ${ }^{120}$. Gemäß der offiziellen Sichtweise galt hinsichtlich der Einzelleitung von nun an: „Dieses Prinzip ... verbietet es, daß andere Organe, ja selbst dienststellungs- und rangmäßig höhere Offiziere, die nicht der Linie nach direkte Vorgesetzte sind, sich in die Belange der militärischen Führung der Einheit, des Truppenteils beziehungsweise Verbandes einmischen, irgendwelche Weisungen erteilen oder Maßnahmen ... treffen." 121

Als die SED diese Änderung knapp anderthalb Jahre nach dem Mauerbau initiierte, befürchtete sie anscheinend nicht mehr, daß die gestärkte Entscheidungsautonomie der militärischen Truppenführung die "führende Rolle" der Partei in der NVA gefährden könnte. Im übrigen hatte die Neubestimmung der Führungsgrundsätze keinen entscheidenden Einfluß auf das Verhältnis der Kommandeure zu den Politorganen und Parteiorganisationen. Die Leiter der Politorgane unterstanden den Kommandeuren nach wie vor nur in der Funktion als Stellvertreter für politische Arbeit. Die Politorgane als parallel zur militärischen Hierarchie bestehender eigenständiger Kontrollapparat fielen jedoch auch weiterhin nicht unter die Befehlsbefugnis der Kommandeure. In ihrer Parteiorganisation erhielten die Kommandeure ebenfalls keinen anderen Status zugesprochen. Der Parteiapparat in der NVA war mittlerweile soweit fortentwickelt worden, daß die SED jederzeit die Möglichkeit hatte, die Entschlußfassung einer militärischen Leitung zu überprüfen und, wenn nötig, zu intervenieren. Trotzdem aber war die formale Stärkung der Stellung der Kommandeure als Einzelleiter mit einem gewissen Zuwachs an Entscheidungskompetenz verbunden: dies galt nicht zuletzt für ihre Aufgaben als Disziplinarvorgesetzte ${ }^{122}$ und in Kaderfragen.

So waren die Disziplinarvorgesetzten gemäß der neuen Disziplinarvorschrift DV-10/6, die am 1. Januar 1963 in Kraft trat, nicht mehr dazu verpflichtet, sich vor der Entscheidung über eine Disziplinarmaßnahme mit dem Politstellvertreter und dem Parteisekretär des zu Bestrafenden zu beraten. Die bis dahin gültige Disziplinar- und Beschwerdeordnung von 1957 hatte diese Verpflichtung enthalten ${ }^{123}$. Ent-

119 Innendienstvorschrift DV-10/3, 1959, S. $16 \mathrm{f}$.

120 Innendienstvorschrift DV-10/3, 1963, S. $31 \mathrm{f}$.

121 Hartmann, Die Abgabe von Straftaten an den Kommandeur, S. 43.

122 Vgl. Herspring, East German Civil-Military Relations, S. 109.

123 Vgl. S. $136 \mathrm{ff}$. 
sprechend den neuen Bestimmungen hatte der Disziplinarvorgesetzte bei einem Verstoß eines NVA-Angehörigen gegen die militärische Disziplin oder die öffentliche Ordnung zu entscheiden, ob er diesen ermahnen sollte, das Vergehen durch ein militärisches Kollektiv zu behandeln oder eine Disziplinarstrafe zu verhängen war ${ }^{124}$. Wie Hoffmann im Mai 1963 konstatierte, war nunmehr „eindeutig“ fixiert worden, „daß der Kommandeur darüber entscheidet, welche Disziplinarmaßnahmen angewandt werden sollen"125. Und auch die Behandlung des Disziplinarverstoßes in einem Kollektiv bedeute nicht, so der Minister, daß der Kommandeur „seine Disziplinarbefugnisse aus der Hand“ gebe ${ }^{126}$. Diese Sicht bestätigte auch die neue Melde- und Untersuchungsordnung vom Dezember 1964, aus der zudem hervorging, daß die Entscheidung, ob es sich bei dem Vergehen eines Untergebenen um einen bloßen Disziplinarverstoß oder aber um eine Straftat handelte, weiterhin vom zuständigen Militärstaatsanwalt, nicht jedoch vom jeweiligen Kommandeur und Disziplinarvorgesetzten selbst gefällt wurde. So heißt es in der Melde- und Untersuchungsordnung: „Hat ein Angehöriger der Nationalen Volksarmee eine ungesetzliche Handlung begangen, die vom Militärstaatsanwalt überprüft wurde, jedoch nur einen Disziplinarverstoß darstellt, obliegt die Erledigung ausschließlich dem Kommandeur." 127

Die Disziplinarvorschrift von 1963 ermöglichte es jedem Disziplinarvorgesetzten, ein Vergehen gegen die militärische Disziplin durch ein militärisches Kollektiv behandeln zu lassen. Diese Neuerung erfolgte in Übereinstimmung mit der neuen rechtspolitischen Linie der SED, die verstärkt auf Formen kollektiver Selbsterziehung als Sanktionsinstrumente setzte ${ }^{128}$. Die Innendienstvorschrift von 1963 bestimmte sogar, daß sich ein Kommandeur bei der Erziehung von Armeeangehörigen, die die „militärische Disziplin oder die öffentliche Ordnung“ verletzt hatten, „weitgehend auf das Kollektiv des Truppenteils bzw. der Einheit" stützen sollte"129. Die Mitglieder dieser Kollektive bestanden jeweils aus Angehörigen derselben Dienstgradgruppe, der auch der Beschuldigte angehörte. Verstöße von Offizieren sollten demnach auf Versammlungen der im Dienstgrad oder der Dienststellung gleichgestellten Offiziere auf Regimentsebene behandelt werden ${ }^{130}$. Die Kollektive hatten entsprechend der Melde- und Untersuchungsordnung von 1964 das „Recht, dem Kommandeur Vorschläge zur Erziehung des Täters zu unterbreiten“. Dabei konnte es sich beispielsweise um eine Entschuldigung beim Geschädigten oder die Wiedergutmachung des eingetretenen Schadens handeln ${ }^{131}$. Dies bedeutete aber

124 Disziplinarvorschrift der Nationalen Volksarmee DV-10/6, 1963, S. 22.

125 Hoffmann, Die Arbeit mit den Kadern - ein Hauptanliegen der Führungstätigkeit. Aus dem Referat auf der I. Kaderkonferenz der Nationalen Volksarmee, 24. Mai 1963, in: Ders., Sozialistische Landesverteidigung, T. 1, S. 25.

126 Ebenda.

127 Ordnung über die Meldung, Untersuchung und Bearbeitung von strafbaren Handlungen, besonderen Vorkommnissen und disziplinaren Verstößen, die Zusammenarbeit mit den Militärjustizorganen sowie über die Einbeziehung der Armeeangehörigen in die Rechtspflege - Melde- und Untersuchungsordnung -, 7. Dezember 1964, BA-MA, VA-01/5622, B1. 81.

128 Vgl. Kap. VIII.3. dieser Studie.

129 Innendienstvorschrift DV-10/3, 1963, S. 33.

130 Disziplinarvorschrift der Nationalen Volksarmee DV-10/6, 1963, S. 22.

131 Ordnung über die Meldung, Untersuchung und Bearbeitung von strafbaren Handlungen, besonderen Vorkommnissen und disziplinaren Verstößen, die Zusammenarbeit mit den Mi- 
offensichtlich nicht, daß die Vorschläge für den Disziplinarvorgesetzten bindend waren. $\mathrm{Zu}$ dieser Frage hieß es später erläuternd: „Es sind eben nur Vorschläge, über die der Kommandeur endgültig entscheidet. Deshalb beeinträchtigen sie keineswegs das Prinzip der militärischen Einzelleitung." Es könne durchaus auch Fälle geben, „in denen der Kommandeur entgegen den Vorschlägen entscheiden“ müsse, nämlich insbesondere dann, wenn sie auf „überspitzten Entscheidungen“ beruhten ${ }^{132}$.

In gewisser Weise stellten die Offizierskollektive eine Wiedereinführung der 1961 aufgelösten Offiziersehrenräte als korporative Institutionen kollektiver Selbstkontrolle dar, da bei ihrer Zusammensetzung primär der Dienstgrad, nicht aber die Parteizugehörigkeit entscheidend war. Da dies aber nicht der Absicht der SED entsprach, sah sich der Chef der Politischen Hauptverwaltung Verner bereits vor dem Inkrafttreten der neuen Disziplinarvorschrift im Dezember 1962 genötigt klarzustellen: „Selbstverständlich werden mit der kollektiven Selbsterziehung in unserer Armee keine neuen Ehrengerichte geschaffen." 133 Zudem ist anzunehmen, daß der Politstellvertreter und der Parteisekretär einer Einheit, sofern sie Offizier waren, über eine Sonderstellung innerhalb eines solchen Offizierskollektivs verfügten, so daß sich eine autonome Selbstkontrolle nicht entfalten konnte.

Auf der Grundlage der Melde- und Untersuchungsordnung wurden den Kommandeuren Mitte der sechziger Jahre durch die Militärstaatsanwälte und Militärgerichte zunehmend auch Straftaten von Militärangehörigen „infolge Geringfügigkeit oder geringer Schuld des Täters" zur Entscheidung übergeben. In einem solchen Fall hatte der Kommandeur „unverzüglich ... seine Entscheidung über die zu ergreifenden Disziplinarmaßnahmen zu treffen“. Gemäß der Bestimmungen war er hierbei formal unabhängig. Er sollte sich jedoch zuvor mit der Leitung der Parteibeziehungsweise FDJ-Grundorganisation beraten ${ }^{134}$. Hier zeigte sich ebenfalls die gestärkte Stellung der Disziplinarvorgesetzten und Kommandeure ${ }^{135}$.

Auch im Hinblick auf Personalentscheidungen gab es im Zeitraum nach 1961 einige organisatorische Veränderungen, die den Kommandeuren mehr Verantwortung zukommen ließen. Bereits vor dem Mauerbau hatte die ZK-Abteilung für Sicherheitsfragen nach einer Überprüfung der Verwaltung Kader im Juni 1960 konstatiert, zur „Sicherung der Einsatzfähigkeit“ der Streitkräfte sei es nötig, „das Prin-

litärjustizorganen sowie über die Einbeziehung der Armeeangehörigen in die Rechtspflege - Melde- und Untersuchungsordnung -, 7. Dezember 1964, BA-MA, VA-01/5622, B1. 83.

132 Hartmann, Die Abgabe von Straftaten an den Kommandeur, S. 83.

133 Ausführungen des Stellvertreters des Ministers für Nationale Verteidigung, Admiral Verner, vor dem Kollegium des Ministeriums für Nationale Verteidigung über den „Entwurf des Erlasses des Staatsrates der DDR über die grundsätzlichen Aufgaben und die Arbeitsweise der Organe der Rechtspflege“, 21. Dezember 1962, SAPMO-BArch, DY 30/IV 2/12/47, Bl. 462.

134 Ordnung über die Meldung, Untersuchung und Bearbeitung von strafbaren Handlungen, besonderen Vorkommnissen und disziplinaren Verstößen, die Zusammenarbeit mit den Militärjustizorganen sowie über die Einbeziehung der Armeeangehörigen in die Rechtspflege - Melde- und Untersuchungsordnung -, 7. Dezember 1964, BA-MA, VA-01/5622, Bl. $81 \mathrm{ff}$.

135 So wurden ihnen 1963 etwa 17 Prozent aller angefallenen Straftaten zur Behandlung nach der Disziplinarvorschrift übergeben. Dieser Anteil erhöhte sich 1964 auf 21 Prozent und betrug 1965 schließlich rund 24 Prozent. Hartmann, Die Abgabe von Straftaten an den Kommandeur, S. 36. 
zip der Einzelleitung auf dem Gebiet der Kaderarbeit voll durchzusetzen"136. Anscheinend war es nach Ansicht der SED-Führung jedoch erst unter den geänderten politischen Rahmenbedingungen nach dem 13. August 1961 möglich, grundlegende Modifikationen einzuleiten. Dies geschah parallel zur Verringerung der Zahl militärischer Nomenklaturkader in den Nomenklaturen der leitenden Parteigremien Mitte 1962 ${ }^{137}$. Auch in der NVA sollte eine Reduzierung der Kadernomenklaturen der militärischen Führung und eine Verlagerung der Verantwortlichkeit auf nachgeordnete Führungsebenen erreicht werden. Die Nomenklatur der NVA wurde entsprechend dieser Absicht auf der Grundlage der „vorläufigen Richtlinie für die Arbeit mit der Kadernomenklatur des ZK der SED" neu geregelt und am 13. April 1962 durch den Befehl Nr. 33/62 des Ministers für Nationale Verteidigung eingeführt ${ }^{138}$.

Das Ergebnis dieser Neuregelung war eine weitere Differenzierung der Kadernomenklatur der NVA. Zusätzlich zu den bereits bestehenden Nomenklaturen der Leitung des Ministeriums für Nationale Verteidigung, der Leitungen der Teilstreitkräfte, Militärbezirke und der Stadtkommandantur Berlin sowie der Kommandeure der Verbände schuf man auch Nomenklaturen der Kommandeure der Militärakademie „Friedrich Engels“ und der Offiziersschulen sowie der Kommandeure der dem Ministerium für Nationale Verteidigung unmittelbar unterstellten Truppenteile139. Allein die Nomenklatur des Chefs der Politischen Verwaltung wurde gestrichen, was vermutlich auf die Bildung der Politischen Hauptverwaltung zurückzuführen war $^{140}$. Spätestens jedoch durch den Beschluß vom 13. April 1964 bestätigte das Sekretariat der Politischen Hauptverwaltung eine neue Nomenklatur ${ }^{141}$. Wie die Verwaltung Kader rückblickend feststellte, wurde durch die Erweiterung der Kadernomenklatur der NVA die Verantwortlichkeit „für viele Dienststellungen[,] die bisher einer höheren Nomenklatur angehörten, den nachgeordneten Chefs bzw. Kommandeuren übertragen" 142 .

Trotz dieser Veränderungen scheinen die Nomenklaturvorgesetzten - vor allem unterhalb der Ebene des Ministeriums - zunächst weiter über keinen großen Spielraum verfügt zu haben, auf Personalentscheidungen über Nomenklaturkader sowie über Offiziere, die keiner Nomenklatur angehörten, Einfluß zu nehmen. Bestimmend bei der operativen Gestaltung der Personalpolitik blieben offenbar die Kaderorgane, deren Vorschläge auch jetzt noch einer „maßgeblichen“ Einflußnahme durch die Politorgane und Parteiorganisationen unterliegen sollten ${ }^{143}$. Die Zustän-

136 Bericht der Brigade des ZK der SED in der Verwaltung Kader des Ministeriums für Nationale Verteidigung, 15. Juni 1960, SAPMO-BArch, DY 30/IV 2/12/22, Bl. 299.

137 Vgl. Kap. VIII.1. dieser Studie.

138 Teilbeitrag der Verwaltung Kader zur Einarbeitung in die Gesamtanalyse und Grundkonzeption der Entwicklung der NVA, 10. März 1967, BA-MA, VA-01/5679, Bl. 158.

139 Durchführungsbestimmung zum Erlaß des Staatsrates der DDR über den aktiven Wehrdienst in der NVA, 13. April 1962, BA-MA, VA-01/8738, Bl. 76.

140 Teilbeitrag der Verwaltung Kader zur Einarbeitung in die Gesamtanalyse und Grundkonzeption der Entwicklung der NVA, 10. März 1967, BA-MA, VA-01/5679, Bl. 159.

141 Ebenda, Bl. 164.

142 Ebenda, Bl. 160.

143 Instruktion für die Parteiorganisationen der SED in der NVA und für die Politorgane der NVA. Protokoll Nr. 39/63 der Sitzung des Politbüros des ZK der SED, 5. November 1963, SAPMO-BArch, DY 30/J IV 2/2/904, Bl. 157, 174. 
digkeit der Kommandeure beschränkte sich darauf, die Vorschläge der Kaderorgane zu bestätigen, obwohl die Nomenklaturvorgesetzten formal dazu berechtigt waren, „personelle Veränderungen nach den gültigen Festlegungen zu entscheiden und Vorschläge" darüber „einzureichen"144.

Angesichts dieser Situation kritisierte Hoffmann auf der Kadertagung im Mai 1963, in der Vergangenheit sei die Verantwortung der Kaderorgane „nicht immer im Rahmen der Einzelleitung gesehen" worden. Es habe Versuche gegeben, zwischen der Verantwortung der Kommandeure und der der Kaderorgane eine "Trennung“ zu machen. Damit werde jedoch die „Verantwortung der Kommandeure als Einzelleiter in unzulässiger Weise herabgemindert". Auch seien die Kaderorgane „keine selbständigen Organe“, weshalb sie "nicht ihre eigene Kaderpolitik betreiben" könnten. Eine solche „Selbständigkeit“, die auch in der Verwaltung Kader „hier und da" noch bestehe, dürfe es nicht geben ${ }^{145}$.

Um diesen Mangel zu beheben, sollten neue Grundsätze für die Arbeit mit der Kadernomenklatur herausgegeben werden. Hoffmann erklärte im April 1964, bei der Überarbeitung der bisherigen Bestimmungen sei darauf zu achten, daß der Kommandeur in bezug auf seine Nomenklaturkader in Zukunft "nicht nur eine Entscheidung“ herbeiführe, sondern daß er auch „voll und ganz für diese verantwortlich" sei ${ }^{146}$. Die Grundsätze sollten daher unter anderem mit dem Ziel modifiziert werden, eine „Differenzierung der Verantwortlichkeiten in der Arbeit mit den Nomenklaturkadern herbeizuführen und die Verantwortung der Nomenklaturvorgesetzten, der unmittelbaren Vorgesetzten und der Kaderorgane klarer abzugrenzen und konkreter zu bestimmen“. Vor allem aber galt es, „das Prinzip der persönlichen Verantwortung“ in der Arbeit mit den Nomenklaturkadern „voll durchzusetzen"147. Kaderentscheidungen sollten zwar weiter auf den Vorarbeiten der Kaderorgane fußen, ihre Gültigkeit jedoch erst durch die direkte Mitwirkung der Nomenklaturvorgesetzten erlangen. Daher wurde festgelegt, daß Personalfragen in den militärischen Leitungen und Stäben „im Kollektiv zu beraten“ waren. Die Entscheidung sollte jedoch „auf der Grundlage des Prinzips der Einzelleitung“ erfolgen ${ }^{148}$. Im Ergebnis waren Nomenklaturvorgesetzte und auch unmittelbare Vorgesetzte dazu berechtigt, selbst „personelle Veränderungen“ von Kadern und Nomenklaturkadern einzureichen. Die Aufgaben der Kaderorgane richteten sich nun vor allem auf die „Erarbeitung von Einschätzungen des Nomenklaturbestandes und von Vorschlägen zur Besetzung der Nomenklaturdienststellungen“"149. Diese Änderungen traten im Rahmen der von der Leitung des Ministeriums für Nationale Verteidigung

144 Durchführungsbestimmung zum Erlaß des Staatsrates der DDR über den aktiven Wehrdienst in der NVA, 13. April 1962, BA-MA, VA-01/8738, Bl. 77.

145 Referat des Ministers für Nationale Verteidigung auf der Kadertagung des Ministeriums für Nationale Verteidigung, 24. Mai 1963, BA-MA, VA-01/13871, Bl. $19 \mathrm{ff}$.

146 Protokoll der Sitzung der Leitung des Ministeriums für Nationale Verteidigung, 21. April 1964, BA-MA, AZN/28111, Bl. 4.

147 Grundsätze für die Arbeit mit der Kadernomenklatur. Protokoll der Sitzung der Leitung des Ministeriums für Nationale Verteidigung, 21. April 1964, BA-MA, AZN/28111, Bl. 43.

148 Ebenda, Bl. 33.

149 Ebenda, Bl. 35. 
am 25. Mai 1964 beschlossenen Neufassung der „Bestimmungen für die Arbeit mit den Kadern in der NVA“ in Kraft ${ }^{150}$.

Eine weitere Versachlichung der Personalpolitik in den Streitkräften bewirkte die Einführung sogenannter Attestationen durch einen Befehl des Ministers für Nationale Verteidigung vom 25. Mai 1964. Von diesem Zeitpunkt an sollten die Offiziere des aktiven Wehrdienstes in der Regel alle drei Jahre im Hinblick auf ihre „politische Haltung und Zuverlässigkeit, die militärischen und spezialfachlichen Qualitäten sowie die persönlich-moralischen Eigenschaften“ eingeschätzt werden. Da die Attestationen von den „unmittelbaren Vorgesetzten “ auszuarbeiten waren ${ }^{151}$ und die Grundlage „sowohl für die Einschätzung des Offiziersbestandes insgesamt, als auch für personelle Veränderungen (Beförderung/Einsatz usw.) des einzelnen Offiziers" bildeten, dürfte sich der Einfluß der militärischen Leiter auf die Kaderentwicklung erheblich erhöht haben ${ }^{152}$. Anschließend waren die Attestationen von speziell gebildeten Kommissionen zu überprüfen und durch den nächsthöheren direkten Vorgesetzten zu bestätigen ${ }^{153}$. Die Beurteilungen standen jedoch auch in dieser Form weiterhin unter der Aufsicht der Partei. Zum einen erfolgte die Erarbeitung der Attestationen unter Mitarbeit des Parteisekretärs, wobei aber ausdrücklich darauf hingewiesen wurde, daß die Attestationen „keinesfalls in Sitzungen der Parteileitungen oder in Versammlungen beraten werden" sollten. Dies hätte die Autorität der Vorgesetzten untergraben können. Zum anderen verwirklichte die Partei ihren Einfluß „durch die Arbeit der Kommissionen“. Denn bis zur Ebene der 'Truppenteile gehörten ihnen die Politstellvertreter und Parteisekretäre an. Ab Verbandsebene aufwärts war der Leiter des Politorgans Mitglied der Kommission des jeweiligen Kommandeurs bzw. Chefs ${ }^{154}$.

Der Chef der Verwaltung Kader sah als ein Ergebnis dieser Neuerungen, daß „in der Einschätzung und Beurteilung unserer Kader eine echte Wende zu verzeichnen ... und eine neue, höhere Qualität erreicht " worden sei. Die Kommandeure und Vorgesetzten seien gezwungen gewesen, „sich gründlicher als bisher mit den ihnen unterstellten Offizieren zu beschäftigen, um eine allseitige und objektive Einschätzung erarbeiten zu können"155. Diese in einen Vorwurf gekleidete Feststellung bewies letztlich aber nur, daß die Kommandeure und Vorgesetzten erst durch die erweiterten Kompetenzen in den Stand versetzt worden waren, bei der Beurteilung und Entwicklung des eigenen Personalbestandes selbst entscheidende Impulse zu geben.

150 Teilbeitrag der Verwaltung Kader zur Einarbeitung in die Gesamtanalyse und Grundkonzeption der Entwicklung der NVA, 10. März 1967, BA-MA, VA-01/5679, Bl. 162.

151 Attestierung der Offiziere der NVA. Befehl Nr. 53/64 des Ministers für Nationale Verteidigung, 25. Mai 1964, BA-MA, VA-01/17198, Bl. 28.

152 Uber einige Aufgaben der Politorgane und Parteiorganisationen bei der Attestierung der Offiziere, 21. Dezember 1964, BA-MA, VA-01/17198, Bl. 101.

153 Attestierung der Offiziere der NVA. Befehl Nr. 53/64 des Ministers für Nationale Verteidigung, 25. Mai 1964, BA-MA, VA-01/17198, Bl. 28.

154 Über einige Aufgaben der Politorgane und Parteiorganisationen bei der Attestierung der Offiziere, 21. Dezember 1964, BA-MA, VA-01/17198, Bl. $99 \mathrm{f}$.

155 Bericht über die Durchführung der Attestierung des Offiziersbestandes der NVA, 15. Dezember 1965, BA-MA, VA-01/17198/1, Bl. 281. 